\title{
Coherent heat patterns revealed by unsupervised classification of Argo temperature profiles in the North Atlantic Ocean
}

\author{
Maze Guillaume ${ }^{1,{ }^{*}}$, Mercier Herle ${ }^{2}$, Fablet Ronan ${ }^{3}$, Tandeo Pierre ${ }^{3}$, Lopez Radcenco Manuel ${ }^{3}$, \\ Lenca Philippe ${ }^{3}$, Feucher Charlène ${ }^{1}$, Le Goff Clement ${ }^{3}$
}

${ }^{1}$ Ifremer, UMR 6523 LOPS, Brest, France

${ }^{2}$ CNRS, UMR 6523 LOPS, Brest, France

${ }^{3}$ Institut Mines-Telecom, Telecom Bretagne, UMR CNRS 6285 Lab-STICC Brest, France

* Corresponding author : Guillaume Maze, email address : gmaze@ifremer.fr

\begin{abstract}
:
A quantitative understanding of the integrated ocean heat content depends on our ability to determine how heat is distributed in the ocean and what are the associated coherent patterns. This study demonstrates how this can be achieved using unsupervised classification of Argo temperature profiles. The classification method used is a Gaussian Mixture Model (GMM) that decomposes the Probability Density Function of a dataset into a weighted sum of Gaussian modes.

It is determined that the North Atlantic Argo dataset of temperature profiles con- tains 8 groups of vertically coherent heat patterns, or classes. Each of the temperature profile classes reveals unique and physically coherent heat distributions along the vertical axis. A key result of this study is that when mapped in space, each of the 8 classes is found to define an oceanic region, even if no spatial information was used in the model determination. The classification result is independent of the location and time of the ARGO profiles.

Two classes show cold anomalies throughout the water column with amplitude decreasing with depth. They are found to be localized in the subpolar gyre and along the poleward flank of the Gulf Stream and North Atlantic Current (NAC). One class has nearly zero anomalies and a large spread throughout the water column. It is found mostly along the NAC. One class has warm anomalies near the surface $(50 \mathrm{~m})$ and cold ones below $200 \mathrm{~m}$. It is found in the tropical/equatorial region. The remaining four classes have warm anomalies throughout the water column, one without depth dependance (in the southeastern part of the subtropical gyre), the other three with clear maximums at different depths $(100 \mathrm{~m}, 400 \mathrm{~m}$ and $1000 \mathrm{~m}$ ). These are found along the southern flank of the North Equatorial Current, the western part of the subtropical gyre and over the West European Basin. These results are robust to both the seasonal variability and to method parameters such as the size of the analyzed domain.
\end{abstract}


Keywords : heat content, classification, North Atlantic, stratification, water mass, thermocline, Argo, pattern

\section{Introduction}

As revealed by in-situ and satellite observations, the ocean has undergone significant changes in the past decades. In particular, since the early 1970 s, the ocean stored $93 \%$ of the excess of heat added to the Earth climatic system by the anthropogenically modified radiative balance at the top of the atmosphere (Stocker et al., 2013). The ocean has also been found to be more stratified (Levitus et al., 2012) and Western Boundary Currents have probably shifted poleward and intensified (Wu et al., 2012; Yang et al., 2016). To understand the drivers of these changes requires a quantitative understanding of the integrated ocean heat content. 
The ocean temperature structure is very complex but a simple first-order description is possible. Near the surface, ocean temperature is primarily driven by air-sea heat fluxes and modulated by horizontal heat mean and eddy transports, especially in Western Boundary Current systems (Kwon et al., 2010). This results in the ocean surface temperature to decrease poleward. However, ocean currents are three-dimensional and redistribute heat at different depths. At mid-latitudes, a negative wind-stress curl forces a downward doming of isopycnal and isothermal surfaces (Vallis, 2006), which results in the temperature at depth, e.g. 500m, to be higher in subtropical gyres than at the equator (Talley et al., 2011). This 3-dimensional redistribution of heat in the ocean makes our ability to identify remarkable heat patterns in the horizontal and vertical plans crucial to the understanding of the integrated ocean heat content.

Along the vertical axis, remarkable patterns may be defined a priori using known water masses such as shallow, intermediate and deep waters or layers such as the mixed and Ekman layers and the permanent thermocline. These patterns can be used to partition horizontal or vertical heat transports (Talley, 2003; McCarthy et al., 2012; Buckley et al., 2014). However, they are not used to partition heat content variability despite efforts to formalize the use of reference surfaces in vertically integrating heat content (Palmer and Haines, 2009). The problematic is that the general lack of clear objective definition for vertical patterns, despite a recent effort with regard to the permanent pycnocline (Feucher et al., 2016), impedes their description, especially over long timescales during which their defining properties can change (e.g. Yang and Wang, 2009; Fiedler, 2010).

In the horizontal plan, remarkable large scale patterns are not defined per se. Simple geographical boxes of fixed size and shape are preferred. One is left with the difficult task to look for relevant boxes to explain the large scale structure and variability of the heat content. Many studies define the subtropical and/or subpolar gyres as rectangular boxes from which box-averaged statistics are computed (e.g. Lozier et al., 2010; Bryden et al., 2014; Häkkinen et al., 2015; Grist et al., 2015). Due to limited availability of historical measurements, one can even find entire region signals to be approximated by a single location dataset (e.g. Curry and McCartney, 2001). Obviously, a serial issue with a rectangular box is that it does not take into account the complex structure of the ocean which is not aligned along latitudes and longitudes. The problematic is that, although it is always possible to use more complex polygons than a rectangle to describe a region (e.g. Barrier et al., 2015), this will be difficult, if even possible to do, if a region has to be bounded by a dynamical structure such as a Western Boundary Current.

To identify remarkable heat patterns in the horizontal and vertical plans, their variability and their climatology thus remains a challenge. In this study, we propose to tackle this problem with a method that belongs to the class of unsupervised classification methods. Classification, or clustering, is a statistical method that groups data into classes, or clusters, according to a given similarity metric.

Profile classification has already been used in oceanographic application but to other means. Hjelmervik and Hjelmervik (2013) used a classification method on in situ profiles to predict the local vertical structure of temperature and salinity at a given location, without surface information. Indeed, it is rather common to predict the interior structure of the ocean based on surface data, such as sea surface height, and a model either based on physical principals (Ponte and Klein, 2013) or on historical local regressions (Guinehut et al., 2012). To do so without a surface information is much more complicated though. Hjelmervik and Hjelmervik (2013) grouped profiles according to their Euclidean distance in a reduced dimensional space for latitude/longitude/ temperature/salinity and derived a prediction model of climatological profiles at a given latitude/longitude location. For the methodology to perform better than a classic box averaging method, they determined that 26 groups of profiles were necessary for the North Atlantic Ocean. They later adapted the method to real-time profile prediction using partial observations, and the number of groups decreased to 18 for the method to perform well (Hjelmervik and Hjelmervik, 2014). A classification based prediction method to fill in gaps in two-dimensional data have also been used for satellite measurements with clear success (Aretxabaleta and Smith, 2013). The work from Hjelmervik and Hjelmervik (2013, 2014) extents this idea to vertical profiles with strong promises.

Classification based prediction methods strive in dealing with non-Gaussian statistics, such as observed in frontal regions (Sura, 2010). However, for our goal, which is to characterize remarkable heat patterns, they suffer from two limitations: (i) they take data latitude and longitude as parameters and (ii) they require a rather large number of classes to perform well. We understand that these requirements are imposed to 
ensure a satisfactory prediction performance. But, here, we are interested in identifying remarkable patterns in vertical temperature profiles, their corresponding regional distributions (if any) and their climatology. Therefore, on the one hand there is no reason to impose data coordinates in the classification. Indeed, we should let the classification reveals the spatial coherence, or lack thereof, of temperature profiles and not impose it. Tandeo et al. (2014) demonstrated how unsupervised mixture modeling can be used to classify sea surface temperature and height anomalies into small scale dynamical modes without using the latitude and longitude of the data. We shall demonstrate in this study that indeed, profile based classes are coherent in space in the North Atlantic. On the other hand, we aim to understand, and therefore reduce, the information contained into a large collection of temperature profiles. Thus, it is crucial for the information to be contained into a limited number of classes. This however will depend on how many remarkable patterns are relevant for a given usage.

The paper is organized as follows: in section 2, the dataset is presented; in section 3, the classification method is introduced as well as the methodology we employed to apply it to the Argo temperature dataset; in section 4, we apply classification to analyze the vertical integral of the heat content and in section 5 , the classification of temperature profiles is performed to reveal the ocean internal heat content structure in the North Atlantic. Last, discussion/ conclusion are drawn in section 6 while appendices provide technical details about optimization (Appendix A) and sensitivity experiments (Appendix B) .

\section{Data}

In this study, we used data from the Argo array. Argo is a real-time global ocean observation network. It consists of about 3000 autonomous profilers randomly distributed in all oceans to observe the large scale open ocean out of the high latitudes and marginal seas. Most of the profilers drift freely at a parking depth around $1000 \mathrm{~m}$ and every 10 days descend down to $2000 \mathrm{~m}$ to then rise up to the surface measuring pressure, temperature and salinity. Once at the surface, profile data are transmitted to data assembly centers by satellite after what profilers descent back to their parking depth and start another 10 day cycle. Argo data are now used routinely in physical oceanography and are key to the observation of the ocean climate (Riser et al., 2016).

The Argo database is a collection of more than 1.5 million temperature and salinity profiles going from the surface to $2000 \mathrm{~m}$, evenly distributed throughout the seasonal cycle and with approximately 1 profile per month per $3^{\circ} \times 3^{\circ}$ cell between 2000 and 2014. We extracted the database in December 2014 from the Coriolis GDAC (Argo, 2014). We selected profiles located in the North Atlantic between the equator and $70 \mathrm{~N}$ and between $90 \mathrm{~W}$ and $0 \mathrm{E}$. The collection was reduced to profiles and measurements with correct quality control flags (1, 2, 5 or 8, following the Argo reference table 2 of the user manual, Carval et al., 2015) between the surface and $1400 \mathrm{~m}$. The depth level of $1400 \mathrm{~m}$ was chosen as a compromise between the total number of profiles (the shallower the larger) in the analyzed dataset and the vertical extent of the analysis. We finally interpolated the data on a regular vertical grid with a $5 \mathrm{~m}$ resolution (the original resolution ranges from less than $10 \mathrm{~m}$ at the surface to $200 \mathrm{~m}$ at the bottom of the profile).

Figure 1-A shows the spatial density of the final collection of 100,684 profiles. The North Atlantic is a well observed basin and the spatial density is such that there are around 30 profiles per $1^{\circ} \times 1^{\circ}$ cell over most of the area. For the period covered by the dataset (180 months), this is about $160 \%$ the initial target of the Argo sampling strategy. We note that the data density is not homogeneous though. The subpolar gyre and the North-East, off the Bay of Biscay are more densely sampled than the Western tropical region. This will be taken into account in the analysis. Figure 1-B shows the temporal sampling of the collection of profiles: there are on average 8,400 profiles per month with no significant seasonal bias. Finally, Fig.1-C shows the entire time series of number of profiles per month in the collection. It steadily increased from 2000 to the end of 2013, with a remarkable doubling in 2002, a 200 profile/month increase in 2006 and a peak at the end of 2012. The 2014 decrease is due to the fraction of profiles still awaiting for delayed mode quality control and not incorporated in the dataset used here.

[Figure 1 about here.] 


\section{Methodology}

Hereafter we motivate and then present our methodology based on Gaussian Mixture Models (GMM). Readers already familiar with classification and GMM may want to move directly to section 3.4. This presentation targets the physical oceanographic community and therefore will favor a pragmatic description to introduce the key elements required to manipulate this statistical tool. The reader is referred to Bilmes (1998) and Bishop (2006) for a detailed GMM description and determination method, and to Hannachi (2007) for an example of its usage in atmospheric dynamics.

Here we are interested in modeling the oceanic heat content structure, i.e. in understanding how, along the water column, heat reservoirs are organized. To do so, we propose to analyze the diversity of vertical temperature profiles by way of automatic identification of recurrent profile patterns throughout the collection of profiles. One can think about this as an extension of the water mass approach (whereby specific levels of the temperature profile are considered and possibly associated with a water mass) toward the analysis of stack of water masses.

[Figure 2 about here.]

Figure 2-A shows 50 superimposed profiles randomly drawn out of the Argo collection in order to illustrate the diversity of possible vertical structures: some profiles are almost linear from the surface to the bottom, others exhibit one or more layers with gradients (thermoclines) or homogeneities (mode waters).

The diversity of profiles within the complete collection can statistically be represented by a probability density function (PDF). The PDF captures the relative likelihood of a profile to take on a given pattern at a given depth. If a pattern is recurrent in the collection, its instances will accumulate and create a peak in the PDF. Similarly, if more than one pattern are recurrent, one will observe a PDF with several peaks.

It is important to note that such a PDF of the collection of profiles is not trivial to visualize nor to compute because it requires to enumerate the number of profiles falling in small bins of profile types, something which cannot be determined at this point. We therefore adopted a simplified representation, noted $\mathrm{PDFz}$, by enumerating the number of temperature data at a given depth falling in small bins of temperatures and by scaling the resulting histogram so that the integral at each depth level goes to 1. This is shown in Fig.2-B (at 4 sample depths) and $\mathrm{C}$ (at all depths). The observed PDFz has several peaks and we note that they are not necessarily connected through the vertical dimension, meaning that patterns at a given depth can be found in profiles with different patterns at other depths. Such complexity makes the collection of profiles strongly heteroscedastic, i.e. to have sub-collections with different statistical properties, in particular their variance. To face this we need: (i) to objectively identify recurrent patterns in the collection of profiles, i.e. PDF peaks and (ii) to describe such patterns with a minimum of information, i.e. to create a simple model of PDF peaks. It happens that GMM strives to do exactly that.

Thus we used an unsupervised classification method called GMM to decompose the PDF of profiles (for which one simplified representation, $\mathrm{PDF}$, was shown in Fig.2-B,C) into a weighted sum of multidimensional Gaussian PDF. This will allows us to identify and model the typical vertical structures represented in the collection of profiles. The method is referred to as: (i) classification because it seeks to classify profiles into sub-collections, or classes, according to their similarity, (ii) fuzzy because it provides the probability for a profile to belong to each of the classes, (iii) unsupervised because no information about each class properties is known a priori, only their PDF family is imposed and (iv) mixture modeling because it provides a model for the PDF of the collection as a weighted sum (hence mixture) of Gaussian PDF.

One key point of our methodology that makes it fundamentally different from previous works (Hjelmervik and Hjelmervik, 2013, 2014) is that we don't use the geographical locations of profiles to help identify groups of similar profiles. This choice was motivated by the idea that there is no reason for the vertical structure of a profile to be unique to a given region. We thus want to determine if profiles with similar vertical structures are also co-localized in space, hence defining physically coherent regions in the ocean by the sole virtue of their similarities. If this is the case, as we shall see, such a model could be used to determine the distribution in space and time of particular stack of water masses, pretty much like water mass properties (e.g. temperature, salinity and possibly biogeochemical tracers and stratification) can be used to localize 
water parcels of that water mass in space and time in order to study its distribution and variability (GarcíaIbáñez et al., 2015).

\subsection{Probability Density Function of profiles}

Let's start by introducing the key ingredient to GMM: a multi-dimensional Normal PDF with mean $\mu$ and covariance $\Sigma$ :

$$
\mathcal{N}(x ; \mu, \Sigma)=\frac{1}{\sqrt{(2 \pi)^{D}|\Sigma|}} \exp \left(-\frac{1}{2}(x-\mu)^{\top} \Sigma^{-1}(x-\mu)\right)
$$

where $|\cdot|$ is the determinant and $\cdot^{\top}$ the transpose operators, $x \in \mathbb{R}^{D \times 1}$ is a profile of the $\mathbf{x} \in \mathbb{R}^{D \times N}$ collection, $\mu \in \mathbb{R}^{D \times 1}$ and $\Sigma \in \mathbb{R}^{D \times D}$. The array $\mathbf{x}$ is the dataset we want to analyze: it is made of $N$ profiles (as columns) of $D$ vertical levels (as rows). Note that Eq.(1) is a scalar (higher or equal to 0, but not bounded by 1) defined for $x$ whatever its number of dimensions. We shall refer to $\mathcal{N}(\mathbf{x} ; \mu, \Sigma)$ as the $\mathbb{R}^{1 \times N}$ ensemble of PDF values obtained by computing Eq.(1) for all profiles of the dataset $\mathbf{x} \in \mathbb{R}^{D \times N}$.

Although in section 4 we start by investigating a simpler uni-dimensional case, it is crucial to note here that we explicitly keep the vertical dimension (through the dimensions of $x, \mu$ and $\Sigma$ ) in the formulation of the PDF Eq.(1) because it is along this dimension that we aim to identify coherent patterns.

Let's take an example using the collection of Argo profile statistics plotted in Fig.2. The collection mean profile (black plain line in Fig.2) is a column vector $\mu \in \mathbb{R}^{D \times 1}$ and the collection standard deviation profile (black dashed lines in Fig.2 that are one standard deviation around the mean at a given depth) is the square root of the diagonal of a covariance matrix $\Sigma \in \mathbb{R}^{D \times D}$. We will keep in mind that the covariance matrix has no reason to be diagonal though, because of the vertically coherent patterns. The point here, is that a Gaussian PDF from D-dimensional data takes into account the structure and the scale of patterns exhibited by a population of profiles with $D$ vertical levels.

In practice, and it is the motivation for this study, the collection of Argo profiles cannot be represented appropriately by a single Normal PDF.

\subsection{Gaussian Mixture Modeling}

The core foundation of a GMM is that any PDF can be described as closely as desired with a model of weighted sum of Gaussian PDF (Anderson and Moore, 1979):

$$
p(\mathbf{x})=\sum_{k=1}^{K} \lambda_{k} \mathcal{N}\left(\mathbf{x} ; \mu_{k}, \Sigma_{k}\right)
$$

where the PDF model has $K$ components, each referred to as $\mathbf{c}=k$, from the same parametric Gaussian family given by $\mathcal{N}\left(\mathbf{x} ; \mu_{k}, \Sigma_{k}\right)$ and being associated with weighting coefficients $\lambda_{k}$. One can show that the component weights must satisfy: $0 \leq \lambda_{k} \leq 1$ and $\sum_{k} \lambda_{k}=1$ (see Bishop, 2006, p. 430). These weights are the prior probability densities $p(\mathbf{c}=k)$ of each component (Bishop, 2006, p 110).

We aim to fit a PDF model of the form Eq.(2) to the observed PDF of the vertical profiles:

$$
\hat{p}(\mathbf{x}) \simeq p(\mathbf{x})=\sum_{k=1}^{K} \widetilde{\lambda}_{k} \mathcal{N}\left(\mathbf{x} ; \widetilde{\mu}_{k}, \widetilde{\Sigma}_{k}\right)
$$

where $\hat{p}(\mathbf{x})$ stands for the observed PDF and tildes on parameters $\left(\lambda_{k}, \mu_{k}, \Sigma_{k}\right)$ stand for their best estimates. Gaussian mixture modeling nails down to an optimization problem that can be tackled by maximizing the likelihood of observed profiles. This optimization is referred to as model training. It is solved with the Expectation-Maximization method (McLachlan and Krishnan, 2007) and was computed using the open source Netlab software (Nabney, 2002). For clarity in this methodology introduction, we provide the details of the model training in Appendix A.

The only input parameter to the model training procedure is the number of mixture components $K$. It is a rather difficult problem to determine automatically the most appropriate number of components. There 
exists different methods that are mostly based on estimating the most probable $K$, or minimizing a given metric such as the mixture entropy or misfit with the observed PDF (Fraley and Raftery, 1998). A popular method is the Bayesian Information Criteria (BIC, Schwarz, 1978). The BIC is an empirical approach of the model probability computed as:

$$
B I C(K)=-2 \mathcal{L}(K)+N_{f}(K) \log (n)
$$

where $\mathcal{L}(K)$ is the log likelihood of the trained model with $K$ classes (see Eq.A.1), $N_{f}(K)=K-1+K D+$ $K D(D-1) / 2$ is the number of independent parameters to be estimated (the sum of the component weights, Gaussian means and covariance matrix elements in the D-dimensional data space) and $n$ is the number of profiles used to train the model. The BIC is empirical because the first r.h.s. term in Eq.(4) decreases as the number of classes $\mathrm{K}$ increases while the second r.h.s. term is a penalty term that increases with $\mathrm{K}$ and thus prevents model overfitting the data. The sum of the two terms is expected to exhibit a minimum for the most appropriate $K$. For our dataset, we found the BIC method to provide insightful guidance only if computed using the minimal number of profiles expected to describe the collection. More details are provided in the section 5. Here, the user will keep in mind that the number $K$ sets a compromise between compression (small $K$ ) and accuracy (large $K$ ) of the GMM and that it is meant to provide a number of classes physically meaningful.

At this point, we thus optimally decomposed the observed PDF into $K$ Gaussian densities representing the $K$ components of the mixture model. Each component is defined by a Gaussian density with parameters $\left(\widetilde{\mu}_{k}, \widetilde{\Sigma}_{k}\right)$ and by a $\widetilde{\lambda}_{k}=p(\mathbf{c}=k)$. A component will gather a group, or class or cluster of profiles similar to each others while being at the same time as much as possible different from profiles of other components (the similarity metric being the Mahalanobis distance from the Gaussian density).

How finally do we determine to which component a profile $x$ resemble most, i.e. how do we classify it ? We compute the posterior probability density of a component $p(\mathbf{c}=k \mid x)$ which, given a prior density $p(\mathbf{c}=k)$, is obtained using Bayes' rule $p(\mathbf{c}=k \mid x) p(x)=p(x \mid \mathbf{c}=k) p(\mathbf{c}=k)$ as:

$$
p(\mathbf{c}=k \mid x)=\frac{\widetilde{\lambda}_{k} \mathcal{N}\left(x ; \widetilde{\mu}_{k}, \widetilde{\Sigma}_{k}\right)}{\sum_{k=1}^{K} \widetilde{\lambda}_{k} \mathcal{N}\left(x ; \widetilde{\mu}_{k}, \widetilde{\Sigma}_{k}\right)}
$$

where we recognize that: (i) the mixture $\operatorname{PDF} p(x)$ is given by Eq.(3) for a profile $x$ in the collection $\mathbf{x}$, (ii) the profile PDF conditioned to one component $p(x \mid \mathbf{c}=k)$ is given by Eq.(1) using the optimized set of parameters $\left(\widetilde{\mu}_{k}, \widetilde{\Sigma}_{k}\right)$, a probability sometimes referred to as the activation, and (iii) as already noted, the component prior density $p(\mathbf{c}=k)$ is the weighting coefficient $\widetilde{\lambda}_{k}$.

The probability density $p(\mathbf{c}=k \mid x)$ defined Eq.(5) is the result to be used to classify a profile. It is interesting to note that there are $K$ posterior values for each profile and that they add to 1 . This is why GMM is referred to as a probabilistic or fuzzy classification method, in opposition to hard ones like KMeans which provide a binary classification (probability is reduced to either 0 or 1, Lloyd, 1982).

To ease visualization or discussion of the classification results, a profile is said attributed to or labeled with the class $k$ for which its posterior is maximum:

$$
\mathcal{C}(\mathbf{x})=\underset{k}{\arg \max }(p(\mathbf{c}=k \mid \mathbf{x}), k=1: K)
$$

One can interpret maximum posterior values as follows. As the posteriors $p(\mathbf{c}=k \mid \mathbf{x})$ range from 0 to 1 , one can imagine the two extreme cases where: (1) the profile perfectly matches one of the class center and $p(\mathbf{c} \mid \mathbf{x}) \rightarrow[1,0, \ldots, 0]$ with $K-1$ null values or (2) the profile does not match any of the classes and $p(\mathbf{c} \mid \mathbf{x})=[1 / K, 1 / K, \ldots, 1 / K]$, i.e. classes are equi-probable. In that latter case one should note that all posteriors cannot converge toward zero, which could have been expected if none of the model classes corresponded to the profile, because they must sum to one. Equi-probability is thus the indication of unconclusive labeling. This simple line of arguments puts some bounds on the maximum posterior value: from $1 / K$ for the worst case scenario of equi-probable classes up to 1 for the best case scenario if one of the class 
is a perfect fit for the profile (never reached though in practice). In this study, we introduce the following labeling metric as:

$$
\mathcal{R}(\mathbf{x})=\left(p_{m}-\frac{1}{K}\right) \frac{K}{K-1}
$$

where $p_{m}=\max (p(\mathbf{c} \mid \mathbf{x}))$ is the maximum posterior value used to attribute a class to a profile. This metric is a simple scaling of the likelihood of the class attributed to a profile. It ranges from 0 for an unlikely labeling (equi-probable label) up to 1 for a virtually certain labeling.

\subsection{Dimensionality reduction}

The main difficulty in training a GMM with oceanic profiles is that the dimension along which we want to extract hidden structures within the dataset, the vertical axis, is large. We have to deal in Argo data with at least 30 to 40 levels at which a parameter is defined. If, like in our case, vertical profiles are interpolated on a regular $5 \mathrm{~m}$ grid, we have no less that 280 levels. This large number of dimensions in the problem fundamentally translates into a large number of parameters to be determined in the Gaussian covariance matrices. How to tackle such a problem is still subject to intense research (e.g. Krishnamurthy, 2011; Azizyan et al., 2014). Although this namely "curse-of-dimensionality" problem typically occurs in datasets where the number of training data (profiles in our case) is small compared to the number of dimensions (vertical levels), here we consider a dimension reduction scheme to improve the computational efficiency and robustness of the GMM training.

To reduce the number of dimensions several methods are available. We use the most popular one: Principal Component Analysis (e.g. Thomson and Emery, 2014, p 335) on which spatio/temporal EOFs are based (Bjornsson and Venegas, 1997). With PCA, we can decompose a dataset $\mathbf{x}(z)$ as:

$$
\mathbf{x}(z)=\sum_{j=1}^{d} \mathbf{P}(z, j) \mathbf{y}(j)
$$

where $\mathbf{P} \in \mathbb{R}^{D \times d}$ and $\mathbf{y} \in \mathbb{R}^{d \times N}$ with $d \leq D$. The first rows of $\mathbf{P}$ contain profiles maximizing the structural variance throughout the collection of profiles. Thus if we choose $d<<D$, we can reduce the number of dimensions of the dataset $\mathbf{x}$ while preserving most of its structure. This decomposition creates a new space where the $N$ profiles are not defined with $D$ vertical level values (the $\mathbf{x}$ array) but with only $d$ ones (the $\mathbf{y}$ array). The transition between one space and the other is done through the matrix $\mathbf{P}$ containing the definition of the new dimensions in the original ones ( $d$ vertical profiles of $D$ levels, the eigenvectors of the covariance matrix $\left.\mathbf{x}^{\top} \mathbf{x}\right)$.

[Figure 3 about here.]

Dimensionality reduction comes at the expense of the amount of details represented in the dataset. To investigate that loss of details, one can reverse Eq.(8) with different values of $d$ and compare the root mean squared difference (RMSD) between the reconstructed and original datasets. This is shown in Fig.3 for $d$ ranging from 0 to 30 . Not surprisingly, for all $d$ values, the RMSD is larger near the surface. This is a typical behavior of the PCA decomposition of vertical profiles (see for instance Fig. 20 in Fukumori and Wunsch, 1991). One can also note small scale features appearing ether at depths or with large $d=20$ or $d=30$. This noisy behavior arises from the interpolation of Argo profiles with an original 20 to $50 \mathrm{~m}$ vertical resolution onto standard depth levels with a $5 \mathrm{~m}$ resolution. This is not problematic here because PCA acts as a small scale filter. We choose $d=11$ (black curve in Fig.3) to obtain a RMSD smaller than $0.5^{\circ} C$ at all depths and smaller than $0.1^{\circ} \mathrm{C}$ below $600 \mathrm{~m}$ (thus preserving $99.88 \%$ of the dataset variance). This level of RMSD is satisfactory to analyze the climatological structure of heat we are interested in. It would take twice that number of dimensions to decrease the RMSD below $0.25^{\circ} \mathrm{C}$ at all depths. Using PCA, we thus reduced the number of dimensions from 280 to 11, i.e. by one order of magnitude. This is a very significant data reduction that considerably improves the computational cost of the classification. 
Note that to compute the decomposition Eq.(8), we centered and standardized the dataset $\mathbf{x}$ along each dimension, i.e. along each vertical level, so that the variance of the upper or surface layers did not dominate over the deeper ones in the definition of the new space. One can ultimately note that in this study, PCA is used to compress the dataset (reduce its number of dimensions), not to investigate the structure of its covariance matrix (that, is left to GMM). So we purposely do not show and analyze profiles and maps of the $\mathbf{P}(z, j)$ and $\mathbf{y}$ matrices for which a physical interpretation would be hard to derive.

\subsection{Processing the irregular Argo dataset}

Overall, we choose to train a GMM on a subset of the full collection of profiles and then to classify the all profiles using the trained model. Motivations for this choice are two fold: (i) computational efficiency and (ii) un-biased structural sampling. The later point is crucial. Indeed, we have shown in section 2 and in Fig. 1 that the collection of Argo profiles is not evenly distributed in space, some regions having almost 100 more profiles than others. This is not an issue per se to train a GMM but we want to ensure that the GMM components will possibly reveal spatial information about the dataset, not its sampling spatial density, i.e. to answer the question of whether class, or group of profiles that resemble each others, have a geographical coherent signature or not.

We thus created a training subset by randomly selecting profiles within the full 100,684 collection so that the training subset had a spatial density of 10 profiles per $2^{\circ} \times 2^{\circ}$ grid cell over at least $75 \%$ of the domain (the training subset has finally 7590 profiles). This value was determined by trials and errors as a compromise between the size of the training set and its domain coverage. If we imposed a too high density of profiles, we were excluding less populated regions (such as the western low-latitudes). If we imposed a too low density of profiles, we were dramatically reducing the size of the training set.

Last, note that this sub-setting is not related to a classic cross-validation analysis, which distinguishes training and test datasets. The sensitivity of the classification to sub-setting is discussed in Appendix B.

\subsection{Procedure}

Now that we have introduced all elements of the analysis, it is time to recap the procedure used to perform the unsupervised classification of Argo temperature profiles with GMM:

- create a training subset from the dataset $\mathbf{x}$ where profiles are homogeneously distributed in space, spare the remaining profiles,

- select the training subset and then:

- center and standardize data at each vertical levels,

- reduce the number of dimensions, i.e. compute the $\mathbf{P}(z, j)$ and $\mathbf{y}(j)$ vectors,

- set a number of classes $K$ (using guidance from BIC Eq.4),

- train a GMM using the reduced-dimension training set, i.e. compute the best set of parameters $\left\{\widetilde{\lambda}_{k}, \widetilde{\mu}_{k}, \widetilde{\Sigma}_{k}, k=1 \ldots K\right\}$ with the EM algorithm and Eqs.(A.3-A.4-A.5)

- for all profiles of the dataset:

- center and standardize data at each vertical levels using training subset mean and standard deviation profiles,

- reduce the number of dimensions, i.e. compute the $\mathbf{y}(j)$ vectors, given $\mathbf{P}(z, j)$,

- classify the reduced-dimension profiles, i.e. compute the posteriors, class labels and robustness metric with Eqs.(5-6-7) given the $\left\{\widetilde{\lambda}_{k}, \widetilde{\mu}_{k}, \widetilde{\Sigma}_{k}, k=1 \ldots K\right\}$.

- synthesize class patterns by computing weighted class quantile statistics (e.g. compute the median profile of class $k$ as the median of all profiles weighted by their activation values for class $k$ given by $\left.\mathcal{N}\left(\mathbf{x} ; \widetilde{\mu}_{k}, \widetilde{\Sigma}_{k}\right)\right)$.

Table 1 summarizes all GMM important variables that we introduced in this section.

[Table 1 about here.] 


\section{Structure of the vertical mean temperature}

[Figure 4 about here.]

Here we analyze the PDF of the vertical mean temperature - a proxy for the heat content - with GMM. In the next section we will investigate the internal structure giving rise to that uni-dimensional synthetic representation of the temperature distribution. We used the $0-1400 \mathrm{~m}$ vertical mean temperature from the Argo dataset. This depth range is limited by the available dataset.

Figure 4 illustrates one of the most important feature of the oceanic temperature structure, namely that heat is not concentrated at low latitudes near the equator but in the mid-latitudes. More precisely, heat concentrates in the subtropical gyre where Fig. 4 shows temperature to be larger than $3^{\circ} \mathrm{C}$ compared to the equatorial band. Note that the dataset is centered around the domain average, about $9^{\circ} C$, thus in the following, anomalies refer to that center.

[Figure 5 about here.]

The PDF of the vertical mean temperature dataset is shown in Fig.5-A as a gray bar plot. A striking feature that was not clearly visible in Fig.4, is the modality of the water column mean temperature dataset. Indeed, values accumulate in 3 distinct regions to create PDF peaks: warm, cold and near-zero. Although we can distinguish other peaks, these three largest ones clearly capture the main structure of the dataset. One can also note that a significant fraction of the dataset values is located between the main peaks.

From a more methodological point of view, one can note that the warm and cold flanks of the PDF have different slopes: the cold flank is much steeper than the warm flank. This is due to the fact that ocean temperature cannot be negative (more precisely colder than the freezing point) and thus the dataset distribution on the negative range of possible values resemble a Rayleigh distribution. This lower bound effect is usually seen in PDF of wind speed time series (e.g. Pavia and O'Brien, 1986). For sake of simplicity, we do not analyze further this limitation of GMM applied to this dataset.

To train a GMM, i.e. to compute the optimized model parameters, on this dataset we need to choose a number of components $K$. Results for the straightforward choice of $K=3$ are given Fig.5-B. The plot represents the most likely 3 Gaussian distributions, Eq.(1) for $k=1: 3$, weighted by their mixture probability (the $\lambda_{k}$ ) so that their sum is the model PDF (cf Eq.(3)), also superimposed on the observed PDF panel A. Class 1 and 3 are distinct and capture the cold and warm peaks. However, the choice of $K=3$ is clearly unsatisfactory to describe the near-zero peak. Class 2 is appropriately centered around the near-zero peak but has a large variance. It thus appears that GMM fills this class with all the data points that are neither in the warm nor in the cold peak regions and consequently loses the ability to properly describe the near-zero peak. This is fundamentally due to the fact that in GMM, data conditional component probabilities cannot be null, or in other words that a data point must be attributed to a class.

We consequently trained a GMM with $K=5$ components. The model PDF is superimposed on the observed PDF in Fig.5-C while details of the decomposition are shown in Fig.5-D. GMM has in this case enough degrees of liberty to characterize all peaks of the observed PDF. Class 1,3 and 5 have small variance and capture the cold, near-zero and warm peaks. The two intermediate classes 2 and 4 , have larger variances. These classes can be seen as transition classes ensuring that the significant amount of dataset values which are not within the peak regions are also classified. This decomposition is rather satisfactory but has yet some limitations. For instance the PDF structure near the $1^{\circ} C$ anomaly range is not captured. One would need to further increase $K$ to reproduce that structure (not shown). This illustrates that the choice for the number of components to impose in training a GMM is left to the operator burden, based on the observed PDF and critic analysis of the GMM results, and on the analysis goal. For instance, to capture the warm and cold peak regions, $K=3$ is a good choice and $K=5$ does not clearly add new information about these peaks.

[Figure 6 about here.] 
Now that we converged on a description of the dataset structure with GMM, we can visualize classes using other information in the database, like data latitude and longitude. Here we simply map data labels from Eq.(6). A map of the labels is shown in Fig. 6 for the $K=5$ GMM. We see that the warm class (label $5)$ coincides with a large zonal band throughout the North-Atlantic subtropical gyre from $15 \mathrm{~N}$ to the Gulf Stream and $45 \mathrm{~N}$ to the East. The cold class (label 1) is confined to a much smaller region in the Labrador Sea and off the coast of the Newfoundland. Interestingly, the near-zero class (label 3) is found in two distinct geographical regions: along the North Atlantic Current and at low latitudes, south of $15 \mathrm{~N}$.

We characterized with GMM components a structure of the dataset that was far from obvious in Fig.4. This analysis shows that the ocean vertical mean temperature distribution - or integral heat content - is much more complex than a simple equator-to-pole meridional gradient. It clearly reveals how ocean dynamics organizes heat in reservoirs embedded between transition regions that may be associated with fronts. If heat would have been uniformly distributed, then the PDF would not have exhibited modality and would have map with similar probability from the equator to the pole. This would have resulted in a series of classes with large overlapping range of temperature anomalies.

\section{Ocean internal heat content structure}

The uni-dimensional analysis conducted in the previous section revealed key features in the distribution of the vertical mean temperature and its associated regional patterns. In this section we aim to determine the internal structure of temperature anomalies giving rise to that integrated point of view and to investigate the corresponding regional distribution.

\subsection{Vertical distribution of heat}

[Figure 7 about here.]

Figure 7-A presents the observed PDFz, i.e. the PDF at each level, of the temperature dataset where data have been centered and standardized at each depth level (which was not the case Fig.2-C). Figure 7-C additionally shows the PDFz at 5, 300, 600 and 1200m depth levels. Like the top 50m of the water column, the surface PDFz does not show clear modes and mostly reflects a meridional gradient of temperature. This is no longer true as we look deeper. From $100 \mathrm{~m}$ to $400 \mathrm{~m}$ depth, three peaks appear centered over cold, near-zero and warm temperature anomalies progressively shifting toward warmer classes. These peaks are very similar to those found for the PDF of the vertical mean temperature (see Fig.5). From 400m to 800m depth, three other peaks can be seen with centres progressively shifting from one anomalous temperature range to another. Below $800 \mathrm{~m}$, a three peaks distribution also emerges with different centres converging toward a narrow range of negative temperature anomalies.

\section{[Figure 8 about here.]}

The purpose of this study is to identify coherent patterns within the collection of profiles that together lead to such a complex PDFz. To do so, we need to train a GMM and first, to determine the number of classes to be used. We used the BIC method presented in the methodological section. For the method to perform appropriately, we found that it is a subset of independent profiles that has to be used to evaluate Eq.(4), not the full or only the training set. Using Argo data, Ninove et al. (2016) provided the most recent estimate of the horizontal correlation scale for temperature as a function of depth. These estimates are appropriate for our study focusing on the climatological temperature structure in the North Atlantic, for which the temporal correlation scale does not have to be taken into account. For the North Atlantic, they found that below $30 \mathrm{~m}$ depth, the horizontal temperature scale is smaller than $200 \mathrm{~km}$ and larger than $100 \mathrm{~km}$, both zonally and meridionally. Thus using a representative value of $150 \mathrm{~km}$ (see their Fig.9), one can expect an approximate number of 900 independent profiles for a $4500 \times 4500 \mathrm{~km}^{2}$ region such as the North Atlantic. As our collection of profiles is far more larger than that, we computed an ensemble of 50 realizations of Eq.(4) using members of 900 random profiles and $\mathrm{K}$ ranging from 1 to 30. The ensemble mean and spread is shown in Fig.8. The BIC clearly exhibits a minimum between $K=5$ and 10, near 7, 8 and 9 . All values 
were tested and evaluated and we settled on $K=8$ as it provided the more physically meaningful results. The sensitivity of the GMM classification to the number of classes is discussed in Appendix B.

[Figure 9 about here.]

The trained GMM defines classes as Gaussian distributions in the reduced dimensional space. To analyze the analytical class structures in that space would be quite complicated and we found much easier to present and discuss classes in the real space along the vertical depth axis. Therefore, we used temperature profiles and their corresponding activation values to compute weighted quantile statistics at each depth level for each class.

Figure 9 shows activation weighted median profiles for each of the classes obtained with $K=8$ in the GMM. This decomposition is the key result of the study and can be described as follows. The largest temperature difference between median class profiles is $22^{\circ} \mathrm{C}$ at the surface, $12^{\circ} \mathrm{C}$ near $-500 \mathrm{~m}, 6^{\circ} \mathrm{C}$ near $-1000 \mathrm{~m}$ and $3^{\circ} \mathrm{C}$ near $-1400 \mathrm{~m}$ (consistently with Fig.2-C). Class 1 is the coldest while four classes (2, 6, 8 and 5) are successively the warmest. Class 1 gathers profiles much colder throughout the water column than the dataset average. The median profile for this class has anomalies colder than $-5^{\circ} \mathrm{C}$ above $-600 \mathrm{~m}$. Class 2 has a much more complex structure. It gathers profiles with cold anomalies below $-100 \mathrm{~m}$ (with the largest amplitude of $-3^{\circ} \mathrm{C}$ around $-500 \mathrm{~m}$ ), no anomalies between -100 and $-200 \mathrm{~m}$ and the largest surface warm anomalies of all classes above $-50 \mathrm{~m}$ (about $7^{\circ} \mathrm{C}$ ). Class 3 on the other hand, resembles class 1 pattern, simply shifted toward the centre of the PDF at all levels. It is a class with decreasing cold anomalies from $-9^{\circ} \mathrm{C}$ near the surface down to less than $-3^{\circ} \mathrm{C}$ below $-400 \mathrm{~m}$. Class 4 is also colder throughout the water column than the dataset average. This class falls within the range of class 1 and 3 median profiles. Class 5 is the warmest class below $-800 \mathrm{~m}$ depth. It is characterized by near neutral conditions above $-400 \mathrm{~m}$ and warm anomalies below $-600 \mathrm{~m}$ with a maximum centered at $-1000 \mathrm{~m}$ of about $3^{\circ} \mathrm{C}$. Class 6 has near neutral conditions below $-400 \mathrm{~m}$ and a warm anomaly maximum of $7^{\circ} \mathrm{C}$ centered at $-100 \mathrm{~m}$, which makes it the warmest class at this depth. Class 7 gathers profiles warmer throughout the water column than the dataset average with anomalies higher than $1^{\circ} \mathrm{C}$ but is never the warmest. Last, class 8 exhibits warm anomalies, higher than $3^{\circ} \mathrm{C}$ above $-800 \mathrm{~m}$, with a clear maximum at $-400 \mathrm{~m}$ of about $5.5^{\circ} \mathrm{C}$ where this class is the warmest of all.

In Figure 9-C, class median profiles are superimposed on the observed PDFz reproduced from Fig.7-A. Figure 7-C and D show the observed and modeled PDFz at 4 selected depth levels and the details of each of the GMM components (this can be seen as equivalent to the details of the uni-dimensional case analyzed section 4 and shown in Fig.5). These plots illustrate how well the modeled PDFz reproduces the observed $\mathrm{PDFz}$ and how each of the $K=8$ classes contribute to the peaks of the PDFz. For instance, one will note: how class 5 reproduces the peak of warm anomalies near $-600 \mathrm{~m}$, how class 6 and 8 contribute to the neutral peak at $-1200 \mathrm{~m}$, how class 8 controls the warm peak of the PDFz near $-300 \mathrm{~m}$ and how class 1 shapes the colder flank of the $\mathrm{PDFz}$ with contribution from class 4 below $-600 \mathrm{~m}$.

[Figure 10 about here.]

More precisely, Fig.10 represents the PDF at each depth of normalized profiles attributed to each of the $K=8$ classes and in each class subtitle is indicated the prior value of classes (the $\lambda_{k}$, also reported Table 2). The prior weighted sum of class PDFz is shown in Fig.7-B to reproduce appropriately the observed one, shown in Fig.7-A. Priors are the densities of the class in the model. They can be seen as the fraction of the dataset that can be attributed to each class. This is confirmed by the explicit computation of that fraction. It is reported on the second line of Table 2. Priors and class fraction in the dataset are very close to each others and indicate for instance, that class 3 gathers $15 \%$ of the profiles collection while class 4 only $8 \%$. This, in fact, is not surprising because priors are computed as the average probabilities of classes within the dataset (Eq.(A.3)). Each of the classes has its own vertical structure, and its relative weight in the complete PDF is not related to its physical relevance as a vertical structure to interpret the integrated heat content. This, in fact, demonstrates one of the key strength of the unsupervised classification method: it can distinguish different recurrent patterns without being biased by those having large amplitudes. 
On the class PDFz shown in Fig.10 are superimposed the 5, 50 and 95\% percentile profiles. These allow us to investigate the vertical structure of the spread (the width of the class PDF at each levels) for each class. Class 1 - which gathers profiles with cold anomalies throughout the water column - has a small spread and the 5-95\% percentile envelope always corresponds to cold anomalies, which basically means that profiles attributed to class 1 are always colder than the dataset average at any level. Class 2 exhibits a moderate temperature spread below $-200 \mathrm{~m}$ where it has negative anomalies for the $5-95 \%$ percentile. Near $-100 \mathrm{~m}$ depth, the spread reaches a maximum and the PDF is positively skewed. Class 3 PDF shows a large spread throughout the water column. This is the class with the largest spread among the GMM. Class 4 has a large spread in the upper layers which decreases downward. Class 5 PDF is narrow around $-600 \mathrm{~m}$ depth with an increasing and large spread downward. Class 6 spread is the largest at its maximum warm anomaly near $-100 \mathrm{~m}$ and is relatively small otherwise. Class 7 spread makes it significantly warmer than the rest of the basin throughout the water column. Last, class 8 is extremely homogeneous near $-300 \mathrm{~m}$ and has a maximum spread around $-700 \mathrm{~m}$.

Figures 9 and 10 focus on statistics for a given class. We now examine statistics for a given profile, i.e. the posteriors (Eq.5). For a given profile, $\mathrm{K}$ posterior values are used to label it, i.e. to attribute it to its most probable class (Eq.6). In the methodology section we introduced a 0 to 1 label metric (Eq.7) from the maximum posterior value in order to determine if, given a model, a profile label can be trusted (maximum posterior close to 1) or should be taken with caution (equi-probable classes with maximum posterior close to $1 / K)$. Table 2 shows the class fractions of profiles having their label metric values in 5 pre-defined ranges (simply following the IPCC likelihood standards). Results are striking: only a very small fraction (no more than $5 \%$ ) of the profiles are unlikely or about as likely as not to be correctly labeled. In other words, the vast majority of the profiles $(88 \%)$ are very likely or virtually certain to be labeled correctly. This truly means that classes are appropriately defined and do not overlap significantly. We note that class 2 profiles have the maximum label likelihood $(92 \%)$ while the minimum is found for the class 4 profiles.

[Table 2 about here.]

\subsection{Relating vertical to horizontal heat distribution}

[Figure 11 about here.]

To generate Fig.9 and Fig.10, we didn't use any geographical information about profiles, nor did we used latitude or longitude in training the GMM. So the question remains: are profiles attributed to each class collocated in space? The answer is provided by Fig.11 where we color-coded profiles with the class they have been attributed to $(\mathcal{C}(\mathbf{x})$, see Eq.6). It is clear that all classes identified with the GMM method with Argo temperature profiles correspond to physically coherent regions. Class 1 covers the subpolar gyre from the Labrador and Irminger Sea to Eastern flank of the Reykjanes Ridge. Class 2 profiles are localized in the equatorial and tropical bands up to $20^{\circ} \mathrm{N}$ to the East and $10^{\circ} \mathrm{N}$ to the West. Class 3 spreads from $45^{\circ} \mathrm{W}$ to the Iceland Basin in the North Atlantic Current (NAC) region. Class 4 profiles are localized on the poleward flank of the Gulf Stream and NAC. Class 5 is centered over the West European Basin down to the Azores Islands. Class 6 covers the tropical band between 10 and $20^{\circ} \mathrm{N}$ on the Southern flank of the North Equatorial Current (NEC). Class 7 is in the South Eastern part of the subtropical gyre between 20 and $35^{\circ} \mathrm{N}$, mostly to the East of $40^{\circ} \mathrm{W}$. Last, class 8 profiles are localized in the Western part of the subtropical gyre, South of the Gulf Stream.

This coherent horizontal distribution of GMM classes provides key indications to interpret the class vertical patterns. The subpolar gyre gathers profiles with relatively uniform cold temperature anomalies throughout the water column (class 1). Low latitudes are regions where heat, positive anomalies in temperature class 2 and 6 , is concentrated in the upper layers while negative anomalies are found at intermediate depth (class 2). These contrasted layers are separated by the equatorial thermocline which explains the spread in the class 2 PDF near $-100 \mathrm{~m}$ depth (Fig.10-B). The fact that class 3 and 4 profiles are observed in frontal regions explains the large spread of possible temperature anomalies because front meanders and meso-scale eddies generated by their instability imply a diversity of possible profiles at the same location. Moreover, class 3 profiles have nearly zero heat content anomalies because they are located between the warm 
and cold reservoirs of the subtropical vs subpolar regions (as illustrated by the vertical heat content integral in Fig.5). Class 5 profiles are clearly located within the sphere of influence of the Mediterranean Outflow. This warm water mass spreads in the North Atlantic near $-1000 \mathrm{~m}$, which explains the deep structure of class 5 profiles. Small spread near $600 \mathrm{~m}$ depth for this class corresponds to the lower Central $11-12^{\circ} \mathrm{C}$ Water (Paillet and Arhan, 1996).

The last two regions are located on the eastern and western parts of the southern subtropical gyre, where the integral heat content is the largest. The horizontal distribution of classes allows us to attribute the class 8 large heat reservoir between 200 and $800 \mathrm{~m}$ depth to the subtropical thermocline and Eighteen Degree Mode Water (EDW, Maze et al., 2009). The EDW thermostad signature can easily be seen Fig.9-A in class 8 between -200 and $-400 \mathrm{~m}$ while the thick permanent thermocline ranges from -400 to $-800 \mathrm{~m}$ below (Feucher et al., 2016). Class 7 localizes a region where heat is near to uniformly distributed through the water column, pretty much like class 1 for cold anomalies.

Last, we examine the geographical distribution of the label metric defined by Eq.(7). This is shown in Fig.12. When compared with Fig.11, the distribution of the label metric seems to primarily indicate that profiles located on the edge of regions delimiting classes are those close to equi-probable labels, i.e. with maximum posterior values near $1 / K$ (see Fig.12-A). However, similar maps produced using only profiles attributed to each class (Fig.12-B to I) reveal a more complex information where robust labels can be found at the edge of class regions when a frontal dynamical structure is involved. For instance, class 8 northern edge exhibits a rather robust labeling (greenish points Fig.12-I) where the Gulf Stream is located (similarly with class 4 south-western profiles). To the opposite, class 5 edges do not correspond clearly with a front and have profile labels with a smaller likelihood all around.

[Figure 12 about here.]

\section{Discussion and conclusion}

In this study we used a Gaussian Mixture Model (GMM) that is an unsupervised classification method (Bishop, 2006) on Argo temperature profiles. Unlike previous studies using such a method (Hjelmervik and Hjelmervik, 2013, 2014) our goal was not to extrapolate a dataset: our goal was to investigate the information contained into a limited number of classes of profiles identified with classification. We have shown that this information was physically meaningful and brought some insight to the oceanic temperature structure.

The GMM was applied to the observed PDF of normalized and compressed temperature profiles. GMM decomposes the observed PDF into a weighted sum of $K$ multi-dimensional Gaussians, the dimension being the vertical axis, using the Expectation-Maximization algorithm. Each Gaussian corresponds to a group of profiles that resembles each others while being as much as possible different from profiles in other groups.

We started the analysis with the classification of $0-1400 \mathrm{~m}$ vertical mean temperatures, a simple unidimensional dataset, which scales as the vertical integral of heat content. In this case, we found that $K=5$ classes was an appropriate choice to capture the structure and modality of the PDF (Fig.5-C). Three of the classes correspond to clear peaks of the PDF for cold, near-zero and warm temperature anomalies with regard to the dataset average. We showed that the cold class is located in the subpolar gyre, the near-zero class mostly at latitudes lower than $10^{\circ} \mathrm{N}$ and the warm class in the western and southern part of the subtropical gyre (Fig.6). The remaining 2 classes identify transition regions between each of the 3 peaks. It is interesting to note that the trivial choice of $K=3$ classes to fit this 3-peak PDF was not the most appropriate. Indeed, as a significant amount of data values were in the transition regions between the peaks, additional Gaussian classes were necessary to fulfill the GMM requirement for each data to be classified.

The uni-dimensional description of the heat content thus reveals a classic meridional distribution. But to non-experts it might be surprising because it is different from a trivial south-to-north gradient of temperature, such as that observed near the surface. There is indeed more heat stored at mid-latitudes than along the equator because of the internal structure of the oceanic stratification. But this internal structure (from which the PDF at each depth is shown in Fig.7-A) cannot be revealed by the simple uni-dimensional analysis. 
Therefore, in a second step, we classified the full temperature profiles using multi-dimensional Gaussians. Given the high dimensionality of the problem, 280 vertical levels, we had to compress the vertical structural information contained in profiles. To do so, we used a standard Principal Component Analysis and reduced that information from 280 temperature values to only 11 eigenvalues for each profile. This reduction preserved $99.88 \%$ of the profile collection structural variance, so that the RMSD between the original and compressed dataset is smaller than $0.5^{\circ} \mathrm{C}$ throughout the water column and smaller than $0.1^{\circ} \mathrm{C}$ below $600 \mathrm{~m}$ (Fig.3).

Within this reduced-dimensional space, we trained a series of GMM and used the BIC for model selection, i.e. to determine the most appropriate number of classes to decompose the PDF. We should point here to the fact that the initial application of this popular method was not satisfactory because the size of the dataset (more than 7000 profiles in the training set) implied a log likelihood much larger than the penalty term which was thus ineffective (see Eq.4). The solution to this issue was to limit the size of the dataset used to compute the BIC to the expected number of independent profiles, 900 for the North Atlantic Ocean climatology. It is thus key to note here that for an application to a dataset with spatio/temporal correlations, one should use data mining methods with caution.

We then detailed results obtained with $K=8$ classes. First, we computed the statistical properties of each classes (activation-weighted profile statistics) and investigated their median and spread vertical structures (Figs.9 and 10). We were able to attribute to each of the classes their features and known water mass or thermocline in a simple way. This part of our study help answer the problematic of the identification of temperature remarkable patterns along the vertical axis. One can also note that two types of classes were found: classes with almost no zero-crossing (class 1, 3,4 and 7) and classes with distinct heat anomaly reservoirs (class 2, 5, 6 and 8).

Furthermore, it is striking to note that the diversity of structures is found mostly in the warm water sphere. Indeed, cold reservoirs from class 1 and 4 have no clear peaks. On the other hand, warm reservoirs from class 5, 6 and 8 exhibit clear maximum in the sub-surface (class 2), intermediate depths (class 8) and at depths (class 5). Class 2 is the only group of profiles with a maximum and a minimum of heat clearly identifiable near the surface and at intermediate depths respectively. On the other hand, class 7 is the only warm group of profiles without a clear vertical structure. The geographic comparison of the vertical mean $K=5$ class (Fig.6) distribution with the full profile $K=8$ class one (Fig.11) further illustrates this point. It can be seen that warm classes $4-5$ of the vertical mean $K=5$ case, decompose into classes 3-5-6-7-8 in the full profile analysis, while cold classes $1-2$ of the vertical mean $K=5$ case remain colocated with classes 1-4 without vertical structure in the full profile analysis. This lack of structure in the cold water sphere is simply due to the fact that ocean loses heat at the surface so that loss of heat, triggering vertical mixing, is similar to a loss of vertical structure. To the opposite, gaining heat at the surface keeps increasing the stratification and create local maxima. The fact that these maxima are not always found at the same depth is due to the horizontal redistribution of heat by currents.

Secondly, we answered the question: are classes of profiles co-localized in space? The answer is yes. When the horizontal distribution of classes was drawn (Fig.11) we found that, although no geographical information was part of the GMM training, each class corresponded to a specific region in the North Atlantic. This key result of our study is far from trivial: it fundamentally means that even if heat circulates and anomalies may be found far from their formation region, the possible vertical stacks of anomalies are unique to a region. In other words, there are no distinct regions in the North Atlantic were the vertical distribution of heat is similar. Note that the vertical structure of the class spread allows us to be more precise: the vertical structure of temperature profiles combine information from water masses (where the spread is small, see for instance class 8 around $-300 \mathrm{~m}$ where the signature of the EDW is found) but also thermoclines or transition layers (where the spread is larger, see for instance in class 2, around -100m, the signature of the equatorial thermocline). So, as the dynamics of the ocean uniquely distributes heat in space, both vertically and horizontally, unsupervised classification of profiles is able to identify the coherent heat patterns arising from that distribution. An analysis of the profile label metric (Fig.12) additionally suggests that horizontal fronts influence the classification probability of a profile. In other words, the probabilistic transition in the horizontal plan from one class to the other is an indication of the presence of a dynamical front.

We tested the sensitivity of the results to the number of classes and to the spatio/temporal sampling 
of the training set (Appendix B). The number of classes reveals a non-linear, or in that case, an nonhierarchical, behavior of the classification: as the number of classes increases, new classes were not simple subsets of parent classes. Using BIC method guidance, the choice of the number of classes will ultimately depend on the user's scientific question and the degree of details necessary. We also found that as long as modes are in the PDF, the classification identifies classes in a coherent way, whether it is from a small or a large region. This is quite an advantage compare to other analysis tools such as EOFs which are domain dependent. Last, we found that our classification of temperature profiles within the North Atlantic Ocean does not depend on the season of the training set. This result, which may be surprising, relates to the fact that classes were found to identify oceanographic natural domains. It means that even if summer and wintertime profiles from the subpolar gyre are different within the thin layer above the seasonal thermocline, they remain more similar to each others than summer or wintertime profiles from another region, like the subtropical gyre for instance. This result also highlights the fact that the classification outcome is related to what is used as the reference of the dataset, in our case the North Atlantic mean temperature profile. A classification of local temperature anomalies for instance, would undoubtedly provide different classification results.

All these results suggest a wide range of possible applications for unsupervised classification of profiles. For instance:

1. Figures 5-6 and 9-11 are highly synthetic benchmarks to validate Ocean General Circulation Models and to analyze climatic projections with regard to horizontal and vertical heat content changes.

2. The geographic distribution of classes reveals in a coherent and elegant way the horizontal extent of oceanographic natural domains such as the subpolar gyre or the western subtropical gyre. This information can be used: (i) to define such domains with interior data only, without using un-natural rectangular boxes as it is usually done, nor surface variables such as Sea Surface Height, (ii) to characterize the horizontal extent variability of a region and (iii) to objectively compare regions between different datasets, in particular those from numerical models.

3. The temporal evolution of the class representative profiles (median or mean) may be used to characterize the interior variability of a region, as an intermediate representation between domain average and local Eulerian anomalies.

4. Separating profiles from one side of a front from the other may reveal useful for data validation and fronts detection.

5. Classifying a profile may help in selecting the appropriate parameters for complex profile-based diagnostics such as the characterization of the permanent pycnocline (Feucher et al., 2016) or simpler ones, like the density threshold used in the determination of the mixed layer depth.

6. The vertical structure of the class spread may be used to detect water masses and thermoclines.

To encourage these applications, the classification model parameters and results are publicly available (http: //dx.doi.org/10.17882/47106, Maze, 2017) and an open source software to handle Profile Classification Model is also distributed (https://forge.ifremer.fr/projects/pcm).

Last, additional improvements to the method used here could be explored. There is in fact no reasons to limit the classification of profiles to temperature data. Salinity could easily be added to investigate the ocean density structure and the role of temperature vs salinity in controlling stratification patterns. Moreover, rather than a classic PCA, more modern methods for dimensionality reduction, such as deep autoencoders (Hinton and Salakhutdinov, 2006) could also be used to feed the GMM classification.

To conclude, we have shown that unsupervised classification of temperature profiles, when not performed in order to complete or fill gaps within a dataset and thus using a limited number of classes and spatial information, can be used to reveal in an intuitive and physically coherent way remarkable heat patterns, their regional distributions and their climatology. It was shown that the possible stack of water masses and thermoclines are unique to a given region in the North Atlantic Ocean. This result can be used to objectively and coherently define the climatology of such regions, and possibly their variability. As an intermediate between domain averaging and local Eulerian analysis, classes of temperature profiles can provide a new framework for the analysis of the heat content climatology and variability and a synthetic 
benchmark to validate Ocean General Circulation Models and to analyze climatic projections with regard to horizontal and vertical heat content changes. Unsupervised classification of profiles is not widely used in physical oceanography and we hope with this study to contribute to demonstrate its potential. More generally, we believe that the increasing amount of data from in-situ observations, typically from Argo and its always increasing number of sensors, will be pivotal in the development of a data-driven model of the structure and variability of the ocean interior.

\section{Acknowledgment}

Argo data used in this study were collected and made freely available by the International Argo Program and the national programs that contribute to it. (http://www.argo.ucsd.edu). The Argo Program is part of the Global Ocean Observing System. This study was supported by an Ifremer grant under the Brittany regional collaboration enhancement program. We thank the editor and anonymous reviewers for their comments, which helped to improve the manuscript. 


\section{Appendix A. GMM training}

GMM is an optimization problem that consists in determining the best estimate for the set of parameters $\theta=\{\lambda, \mu, \Sigma\}$ to minimize the misfit between the PDF of the model and the PDF of observations. Formally, this is stated as the maximization of the log-likelihood of observed profiles conditioned to the model parameters. The log-likelihood of the dataset, assuming independent observations, is:

$$
\mathcal{L}(\theta)=\sum_{i=1}^{N} \log p\left(x_{i} ; \theta\right)
$$

where it is explicit that the log-likelihood is a function of the set of parameters $\theta$, not the dataset, and where $p\left(x_{i} ; \theta\right)$ is the probability given Eq.(2) for the dataset instance $x_{i}$ using parameters $\theta$.

To train a GMM, i.e. to maximize $\mathcal{L}(\theta)$ with regard to $\theta$, we need a dataset $\mathbf{x}$ and a given number of components $K$. Several methods exist to determine the optimum parameters but the most popular is certainly the Expectation-Maximization (EM) algorithm (McLachlan and Krishnan, 2007). It is an iterative procedure that consists in looping through a two-step procedure that is guaranteed to increase the likelihood after each iteration (Dempster et al., 1977). The procedure is stopped once convergence in the parameters or likelihood values is reached. At iteration $n$, the two steps of the procedure are:

- the Expectation-step where one computes the posteriors $p\left(\mathbf{c}=k \mid \mathbf{x} ; \theta^{n}\right)$ for all the profiles of the dataset using the $n^{\text {th }}$ iteration parameter values $\theta^{n}$. Note that the dependence to model parameters is made explicit compared to Eq.(5).

- the Maximization-step which update model parameters $\theta^{n}$ so that they maximize the log-likelihood of the dataset while considering the imposed constrains on the $\lambda_{k}$.

The Maximization-step comes to maximize with regard to each of the model parameters the following function:

$$
J(\lambda, \mu, \Sigma)=\sum_{i=1}^{N} \log \left(\sum_{j=1}^{K} \lambda_{j} p\left(x_{i} ; \mu_{j}, \Sigma_{j}\right)\right)-\omega\left(1-\sum_{j=1}^{K} \lambda_{j}\right)
$$

where $\omega$ is a Lagrange multiplier introduced to consider constraints on mixing coefficients. The detailed derivation of the optimum solution to Eq.(A.2) is beyond the scope of this introduction to GMM. It comes to the following updates, where one may recognize weighted versions of classical Maximum Likelihood estimates:

$$
\begin{aligned}
\lambda_{k}^{n+1} & =\frac{1}{N} \sum_{i=1}^{N} p\left(\mathbf{c}=k \mid x_{i} ; \theta^{n}\right) \\
\mu_{k}^{n+1} & =\frac{\sum_{i=1}^{N} p\left(\mathbf{c}=k \mid x_{i} ; \theta^{n}\right) x_{i}}{\sum_{i=1}^{N} p\left(\mathbf{c}_{\mathbf{k}} \mid x_{i} ; \theta^{n}\right)} \\
\Sigma_{k}^{n+1} & =\frac{\sum_{i=1}^{N} p\left(\mathbf{c}=k \mid x_{i} ; \theta^{n}\right)\left(x_{i}-\mu_{k}^{n+1}\right)\left(x_{i}-\mu_{k}^{n+1}\right)^{\top}}{\sum_{i=1}^{N} p\left(\mathbf{c}=k \mid x_{i} ; \theta^{n}\right)}
\end{aligned}
$$

The model probability of the $k^{t h}$ component $\lambda_{k}^{n+1}$ is the dataset averaged probability of that component (given by the $p\left(\mathbf{c}=k \mid x_{i} ; \theta^{n}\right)$ posterior values). The center $\mu_{k}^{n+1}$ and covariance $\Sigma_{k}^{n+1}$ of the $k^{t h}$ component are given by the data mean and covariance weighted by the posteriors.

All computations were conducted on a desktop computer with Matlab software and the open source Netlab library (Nabney, 2002). 


\section{Appendix B. Sensitivity analysis}

\section{Appendix B.1. Sensitivity to the number of classes}

[Figure 13 about here.]

We have shown in the previous section and Fig.8 that a classic method such as the BIC can be used to determine the number of classes to train a GMM of profiles. However, the spread of the estimate does not provide a distinct single value for the best $\mathrm{K}$ choice, only useful guidance within a small range of possible values between 5 and 10. Therefore, it is in fact the user who will ultimately decide what is the most appropriate $K$ for a given application.

As an indication, Figure B.13 shows the map of labels for an increasing number of components (from 3 to 9 , case 8 was shown in Fig.11). For $K=3$ (and 2, not shown), the classification is mostly meridional and somehow captures the uni-dimensional analysis information discussed section in 4 . For larger $K$, Eastern and Western regions appear distinctly. It may be noted that the classification is not hierarchical in a sense that for $K$ larger than 4, one class is not necessarily a subset of a larger parent class. For instance, class 1 for $K=6$ (Fig.B.13-D) is a mixed of some of the profiles from class 2,3 and 4 for $K=5$ (Fig.B.13-C).

In the previous section, we choose $K=8$ because it refined the $K=7$ description of the NAC and subpolar gyre while avoiding the $K=9$ split of the equatorial region and thermocline vertical structure into 2 distinct eastern and western classes.

Last, one may note that even if the number of classes increases, some regions remain unchanged and identified in a distinct class. This is particularly true for 4 regions: the subpolar gyre, the western subtropical gyre south of the Gulf Stream, the Mediterranean outflow region and the lowest latitude band. This is already apparent with $K=4$ classes. This basically means that the core information of the climatological distribution of heat in the North Atlantic is: cold anomalies throughout the vertical in the subpolar gyre, warm anomalies near the surface and cold anomalies at depth at low latitudes and warm anomalies in the upper-mid depth in the western and lower mid-depth is the eastern side of the subtropical gyre. This may sound trivial in terms of descriptive oceanography, except that here the structure was elegantly and objectively revealed by unsupervised classification of profiles. The detailed analysis for $K=8$ conducted in the previous section provided additional details to refine this big picture.

\section{Appendix B.2. Sensitivity to the domain and seasonal sampling}

One can also wonder how sensitive is the classification to the geographical extent of the training set. To investigate this issue, we defined a series of rectangular sub-domains and, using the reference GMM classification map (Fig.11), we determined the expected number of classes to be identified in each subdomain. We then used these number of classes to train different GMMs on each of the sub-domains and compared the classification map to the reference case. Results are shown in Fig.B.14 for 6 sub-domains covering different regions and having different spatial scales, both meridionally and zonally. We found that for each sub-domain, the classification map reproduces the expected geographical distribution of labels when compared to the reference case. We conclude that for an appropriate choice of the number of classes, the GMM classification results are not particularly sensitive to the geographical extent of the training set. But one should not forget, that this will be verified as long as the sub-domain PDF will contain the modes to be identified by GMM.

\section{[Figure 14 about here.]}

We furthermore investigated the sensitivity of the classification results to the seasonal sampling of the training set. Indeed, as we used the entire collection of Argo profiles without distinction on the time of sampling, one can wonder if the classification results are not biased toward a particular season or if, for instance, deeper mixed layers from the winter time would lead to another classification outcome. We trained 4 new GMMs using the 4 subsets of profiles sampled in the four seasons: December/January/February, etc... Using $K=8$, the 4 seasonal classifications (not shown) produce classes and maps of labels very similar to those from the complete dataset (Figs.9 and 11). It is probable that using a much significant number of classes would provide GMM the ability to distinguish seasonal signals in the classification outcome. 


\section{References}

Anderson, B. D., Moore, J. B., 1979. Optimal filtering. 1979.

Aretxabaleta, A. L., Smith, K. W., 6 2013. Multi-regime non-gaussian data filling for incomplete ocean datasets. Journal of Marine Systems 119-120 (0), 11-18.

http://www.sciencedirect.com/science/article/pii/S0924796313000493

Argo, 2014. Argo float data and metadata from global data assembly centre (Argo GDAC) - snapshot of Argo GDAC as of December, 8th 2014. http://doi.org/10.17882/42182

Azizyan, M., Singh, A., Wasserman, L., 2014. Efficient sparse clustering of high-dimensional non-spherical gaussian mixtures. arXiv preprint arXiv:1406.2206.

Barrier, N., Deshayes, J., Treguier, A.-M., Cassou, C., 1 2015. Heat budget in the north atlantic subpolar gyre: Impacts of atmospheric weather regimes on the 1995 warming event. Progress in Oceanography 130, 75-90. http://www.sciencedirect.com/science/article/pii/S0079661114001645

Bilmes, J. A., 1998. A gentle tutorial of the em algorithm and its application to parameter estimation for gaussian mixture and hidden markov models. International Computer Science Institute.

Bishop, C. M., 2006. Pattern recognition and machine learning. Springer, New York.

Bjornsson, H., Venegas, S. A., 1997. A Manual for EOF and SVD Analyses of Climatic Data. Center for Climate and Global Change Research.

Bryden, H. L., King, B. A., McCarthy, G. D., McDonagh, E. L., 2014. Impact of a 30\% reduction in atlantic meridional overturning during 2009-2010. Ocean Science Discussions 11 (2), 789-810.

http://www.ocean-sci-discuss.net/11/789/2014/

Buckley, M. W., Ponte, R. M., Forget, G., Heimbach, P., 2014. Low-frequency sst and upper-ocean heat content variability in the north atlantic. Journal of Climate.

http://dx.doi.org/10.1175/JCLI-D-13-00316.1

Carval, T., Keeley, R., Takatsuki, Y., Yoshida, T., Schmid, C., Goldsmith, R., Wong, A., Thresher, A., Tran, A., Loch, S., Mccreadie, R., Argo Data Management Team, 12 2015. Argo user's manual v3.2. Tech. rep., Ifremer.

http://archimer.ifremer.fr/doc/00187/29825/

Curry, R. G., McCartney, M. S., 2001. Ocean gyre circulation changes associated with the North Atlantic oscillation. J. Phys. Oceanogr. 31 (12), 3374-3400.

Dempster, A. P., Laird, N. M., Rubin, D. B., 1977. Maximum likelihood from incomplete data via the em algorithm. Journal of the Royal Statistical Society. Series B (Methodological) 39 (1), 1-38.

http://www.jstor.org/stable/2984875

Feucher, C., Maze, G., Mercier, H., 2016/04/15 2016. Mean structure of the north atlantic subtropical permanent pycnocline from in-situ observations. Journal of Atmospheric and Oceanic Technology. http://dx.doi.org/10.1175/JTECH-D-15-0192.1

Fiedler, P. C., 2010. Comparison of objective descriptions of the thermocline. Limnol. Oceanogr. Methods 8, 313-325.

Fraley, C., Raftery, A. E., 01 1998. How many clusters? which clustering method? answers via model-based cluster analysis. The Computer Journal 41 (8), 578-588.

http://comjnl.oxfordjournals.org/content/41/8/578

Fukumori, I., Wunsch, C., 1991. Efficient representation of the north atlantic hydrographic and chemical distributions. Progress in Oceanography $27(1-2), 111-195$. http://www.sciencedirect.com/science/article/pii/007966119190015E

García-Ibáñez, M. I., Pardo, P. C., Carracedo, L. I., Mercier, H., Lherminier, P., Ríos, A. F., Pérez, F. F., 6 2015. Structure, transports and transformations of the water masses in the atlantic subpolar gyre. Progress in Oceanography 135, 18-36. http://www.sciencedirect.com/science/article/pii/S0079661115000506

Grist, J., Josey, S., Jacobs, Z., Marsh, R., Sinha, B., Van Sebille, E., 2015. Extreme air-sea interaction over the north atlantic subpolar gyre during the winter of 2013-2014 and its sub-surface legacy, 1-19. http://dx.doi.org/10.1007/s00382-015-2819-3

Guinehut, S., Dhomps, A.-L., Larnicol, G., Le Traon, P.-Y., 2012. High resolution 3-d temperature and salinity fields derived from in situ and satellite observations. Ocean Science 8 (5), 845-857. http://www . ocean-sci.net/8/845/2012/

Häkkinen, S., Rhines, P. B., Worthen, D. L., 2015. Heat content variability in the north atlantic ocean in ocean reanalyses. Geophysical Research Letters, 2015GL063299. http://dx.doi.org/10.1002/2015GL063299

Hannachi, A., 2015/01/07 2007. Tropospheric planetary wave dynamics and mixture modeling: Two preferred regimes and a regime shift. Journal of the Atmospheric Sciences 64 (10), 3521-3541. http://dx.doi.org/10.1175/JAS4045.1

Hinton, G. E., Salakhutdinov, R. R., 2006. Reducing the dimensionality of data with neural networks. Science 313 (5786), 504-507.

Hjelmervik, K., Hjelmervik, K., 2013. Estimating temperature and salinity profiles using empirical orthogonal functions and clustering on historical measurements. Ocean Dynamics 63 (7), 809-821. http://dx.doi.org/10.1007/s10236-013-0623-3

Hjelmervik, K., Hjelmervik, K., 2014. Time-calibrated estimates of oceanographic profiles using empirical orthogonal functions 
and clustering. Ocean Dynamics 64 (5), 655-665.

http://dx.doi.org/10.1007/s10236-014-0704-y

Krishnamurthy, A., 2011. High-dimensional clustering with sparse gaussian mixture models.

Kwon, Y.-O., Alexander, M. A., Bond, N. A., Frankignoul, C., Nakamura, H., Qiu, B., Thompson, L. A., 2010. Role of the gulf stream and kuroshio-oyashio systems in large-scale atmosphere-ocean interaction: A review. Journal of Climate 23 (12), 3249-3281.

http://dx.doi.org/10.1175/2010JCLI3343.1

Levitus, S., Antonov, J. I., Boyer, T. P., Baranova, O. K., Garcia, H. E., Locarnini, R. A., Mishonov, A. V., Reagan, J. R., Seidov, D., Yarosh, E. S., Zweng, M. M., 2012. World ocean heat content and thermosteric sea level change (0-2000 m), 1955-2010. Geophysical Research Letters 39 (10), n/a-n/a. http://dx.doi.org/10.1029/2012GL051106

Lloyd, S., 1982. Least squares quantization in pcm. IEEE Transactions on Information Theory 28 (2), $129-137$.

Lozier, M. S., Roussenov, V., Reed, M. S. C., Williams, R. G., 10 2010. Opposing decadal changes for the north atlantic meridional overturning circulation. Nature Geosci 3 (10), 728-734. http://dx.doi.org/10.1038/ngeo947

Maze, G., 2017. A profile classification model from North-Atlantic Argo temperature data. SEANOE. http://dx.doi.org/10.17882/47106

Maze, G., Forget, G., Buckley, M., Marshall, J., Cerovecki, I., 2009. Using transformation and formation maps to study the role of air-sea heat fluxes in north atlantic eighteen degree water formation. Journal of Physical Oceanography 39 (8), $1818-1835$. http://dx.doi.org/10.1175\%2F2009JP03985.1

McCarthy, G., Frajka-Williams, E., Johns, W. E., Baringer, M. O., Meinen, C. S., Bryden, H. L., Rayner, D., Duchez, A., Roberts, C., Cunningham, S. A., 2012. Observed interannual variability of the atlantic meridional overturning circulation at $26.5^{\circ} \mathrm{n}$. Geophysical Research Letters 39 (19). http://dx.doi.org/10.1029/2012GL052933

McLachlan, G., Krishnan, T., 2007. The EM algorithm and extensions. Vol. 382. John Wiley \& Sons.

Nabney, I., 2002. NETLAB: algorithms for pattern recognition. Springer Science \& Business Media. http://www . aston.ac.uk/eas/research/groups/ncrg/resources/netlab/

Ninove, F., Le Traon, P.-Y., Remy, E., Guinehut, S., 2016. Spatial scales of temperature and salinity variability estimated from argo observations. Ocean Science 12 (1), 1-7. http://archimer.ifremer.fr/doc/00317/42845/

Paillet, J., Arhan, M., 1996. Shallow pycnoclines and mode water subduction in the Eastern North Atlantic. J. Phys. Oceanogr. 26 (1), 96-114.

Palmer, M. D., Haines, K., 2009. Estimating oceanic heat content change using isotherms. Journal of Climate 22 (19), 49534969.

http://dx.doi.org/10.1175/2009JCLI2823.1

Pavia, E. G., O'Brien, J. J., 2016/01/12 1986. Weibull statistics of wind speed over the ocean. Journal of Climate and Applied Meteorology 25 (10), 1324-1332. http://dx.doi.org/10.1175/1520-0450(1986)025<1324:WSOWSO>2.0.CO;2

Ponte, A., Klein, P., 2013. Reconstruction of the upper ocean 3d dynamics from high-resolution sea surface height. Ocean Dynamics 63 (7), 777-791. http://dx.doi.org/10.1007/s10236-013-0611-7

Riser, S. C., Freeland, H. J., Roemmich, D., Wijffels, S., Troisi, A., Belbeoch, M., Gilbert, D., Xu, J., Pouliquen, S., Thresher, A., Le Traon, P.-Y., Maze, G., Klein, B., Ravichandran, M., Grant, F., Poulain, P.-M., Suga, T., Lim, B., Sterl, A., Sutton, P., Mork, K.-A., Velez-Belchi, P. J., Ansorge, I., King, B., Turton, J., Baringer, M., Jayne, S. R., 02 2016. Fifteen years of ocean observations with the global argo array. Nature Clim. Change 6 (2), 145-153. http://dx.doi.org/10.1038/nclimate2872

Schwarz, G., 03 1978. Estimating the dimension of a model. Ann. Statist. 6 (2), 461-464. http://dx.doi.org/10.1214/aos/1176344136

Stocker, T., Qin, D., Plattner, G. K., Tignor, M., Allen, S. K., Boschung, A., Nauels, A., Xia, Y., Bex, V., Midgley, M., 2013. The physical science basis. In: Climate Change 2013. Working Group I Contribution to the Fifth Assessment Report of the IPCC. Cambridge University Press.

Sura, P., 2010. On non-gaussian sst variability in the gulf stream and other strong currents 60 (1), 155-170. http://dx.doi.org/10.1007/s10236-009-0255-9

Talley, L. D., 2003. Shallow, intermediate and deep overturning components of the global heat budget. Journal Of Physical Oceanography $33,530-560$.

Talley, L. D., Pickard, G. L., Emery, W. J., Swift, J. H., 2011. Chapter 4 - typical distributions of water characteristics. In: Swift, L. D. T. L. P. J. E. H. (Ed.), Descriptive Physical Oceanography (Sixth Edition), sixth edition Edition. Academic Press, Boston, pp. $67-110$. http://www.sciencedirect.com/science/article/pii/B9780750645522100046

Tandeo, P., Chapron, B., Ba, S., Autret, E., Fablet, R., July 2014. Segmentation of mesoscale ocean surface dynamics using satellite sst and ssh observations. Geoscience and Remote Sensing, IEEE Transactions on 52 (7), 4227-4235.

Thomson, R. E., Emery, W., 2014. Data analysis methods in physical oceanography. Newnes.

Vallis, G. K., 2006. Atmospheric and Oceanic Fluid Dynamics, fundamentals and large-scale circulation. Cambridge University Press.

Wu, L., Cai, W., Zhang, L., Nakamura, H., Timmermann, A., Joyce, T., McPhaden, M. J., Alexander, M., Qiu, B., Visbeck, 
M., Chang, P., Giese, B., 03 2012. Enhanced warming over the global subtropical western boundary currents. Nature Clim. Change 2 (3), 161-166.

http://dx.doi.org/10.1038/nclimate1353

Yang, H., Lohmann, G., Wei, W., Dima, M., Ionita, M., Liu, J., 2016. Intensification and poleward shift of subtropical western boundary currents in a warming climate. Journal of Geophysical Research: Oceans, n/a-n/a. http://dx.doi.org/10.1002/2015JC011513

Yang, H., Wang, F., 2009. Revisiting the thermocline depth in the equatorial pacific. Journal of Climate 22 (13), $3856-3863$. http://dx.doi.org/10.1175/2009JCLI2836.1 


\section{List of Figures}

1 Spatio/temporal sampling of the 100,684 temperature profiles in the Argo dataset used in this study. . . . . . . . . . . . . . . . . . . . . .

2 Plot A: 50 profiles randomly drawn out of the Argo collection. Plot B: Sample of PDFz at depth levels: 5, 300, 600 and 1200m. Plot C: All observed PDFz, i.e. the PDF of temperature at each vertical levels, here computed using the entire collection. The colorscale is the PDF value so that the integral of one PDFz goes to 1 . On plots $\mathrm{A}$ and $\mathrm{C}$ are superimposed in black the mean (plain) and mean \pm one standard deviation (dashed) at each depth. On plot C, horizontal dashed lines indicate plot B selection of PDFz. . . . . . . . . . . . .

3 Centered Root Mean Squared Difference between the reconstructed and original datasets of temperature for an increasing number of dimensions used during dimensionality reduction with PCA. The plot can be read as follows: it takes 11 reduced-dimensions (black curve) to compress the dataset in a way that the RMSD is lower than $0.5^{\circ} \mathrm{C}$ throughout the water column. Vertical tick marks are centered at $0.0125,0.025,0.05,0.1,0.25,0.5$ and $1{ }^{\circ} C . \ldots$

4 Horizontal distribution of the $0-1400 \mathrm{~m}$ vertical mean temperature, plotted as anomalies with regard to the domain average (which is $9^{\circ} \mathrm{C}$ ). We want to investigate the vertical structure of heat anomalies leading to such pattern. Note that only $7.5 \%$ of the profiles have been used to generate this plot (roughly 10 profiles per $2 \times 2$ grid cell) in order to limit overlapping dot

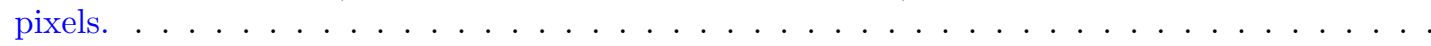

5 Left plots: gray bars are the observed PDF of the uni-dimensional dataset represented Fig.4 and thick black lines are the GMM PDFs for top: $K=3$ components and bottom: $K=5$ components. Right plots: Decomposition of GMM PDFs into their 3 and 5 components. Colored PDFs are the prior weighted activations of each component. Three distinct modes are clearly visible in the dataset (at the extreme and center of the complete PDF) and are nicely captured by GMM. . . . . . . . . . . . . . . . . . . . .

6 Map of profiles color-coded with the class they have been attributed by Eq.(6) for the unidimensional analysis of vertical mean temperature using a GMM with $K=5$. Class colorcodes are similar to those from Fig.5-D. . . . . . . . . . . . . . . . . . . .

$7 \quad$ Plot A: Observed PDFz (PDF at each vertical levels) of profiles from the normalized (centered and standardized) temperature dataset. Plot B: Model PDFz from the prior weighted sum of class PDFz (shown in Fig.10). Plot C: Observed (gray bars) and model (thick black line) PDFz for selected depth levels: 5, 300, 600 and 1200m. Plot D: Decomposition of the model $\mathrm{PDF}$ for the same selected depth levels. Colored lines are the prior weighted activations of each class, following the color convention used Fig.9. . . . . . . . . . . . . . .

8 Ensemble mean and spread ( \pm one standard deviation) of the Bayesian information criteria (BIC) for an increasing number of GMM classes K. The ensemble was computed using 50 members of 900 independent profiles with Eq.(4) . . . . . . . . . . . . . . .

$9 \quad$ Activation-weighted median profiles for each classes. Plot A: with real temperature profiles (the sample mean reference profile is in black), plot B: with profiles of temperature anomalies (centered dataset) and plot $\mathrm{C}$ with profiles of normalized temperature anomalies (level centered/standardized dataset). Grey shading in plot $\mathrm{C}$ is the observed $\mathrm{PDFz}$ of the dataset (reproduced from Fig. 7-A). . . . . . . . . . . . . . . . . . . .

10 Normalized temperature anomaly for each class as a function of depth: black dashed lines are the 5, 50 and 95\% percentiles of the class (note that the $50 \%$ percentile is the median profile plotted in Fig.9). Grey shading is the corresponding PDFz for each class temperature anomalies. Also given in the plot title are the prior values $\lambda_{k}$ from Eq.(A.3) of the class. Statistics were computed using temperature data weighted by activations. . . . . . . . . .

11 Map of profiles color-coded with the class they have been attributed to with Eq.(6) by the classification of temperature profiles using a GMM with $K=8$. Only profiles with a label metric higher than $90 \%$ (Eq.7) with a limit of 10 profiles per $2 \times 2$ grid cell have been used in order to limit overlapping dot pixels. . . . . . . . . . . . . . . . . . . 
12 Map of profiles color-coded with their label metric from Eq.(7) for the classification of temperature profiles using a GMM with $K=8$. See how labels are less robust along the edges of classes shown in Fig.11. Map A is for all profiles while maps B to I are for profiles attributed to class 1 to 8 respectively. No more than 10 profiles per $2 \times 2$ grid cell have been plotted in order to limit overlapping dot pixels. . . . . . . . . . . . . . . . . . . . . 30

B.13 Map of labels obtained with different number of classes $K$. Map A corresponds to $K=3$, map B to $K=4$, map $\mathrm{C}$ to $K=5$, map D to $K=6$, map E to $K=7$ and map $\mathrm{F}$ to $K=9(K=8$ is shown

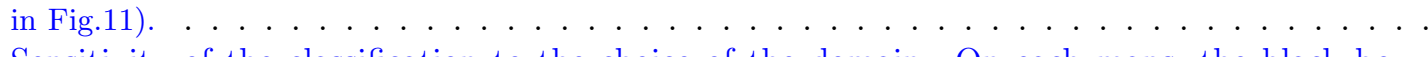

B.14 Sensitivity of the classification to the choice of the domain. On each maps, the black box indicates the domain used to train a new GMM, and profiles are color-coded with the class they have been attributed to. Profiles not used in the GMM training are simply shown as black dots. Note that class colors are arbitrary and only the relative distribution of classes is to be compared with the reference case shown in Fig.11. . . . . . . . . . . . . . . . . 4 

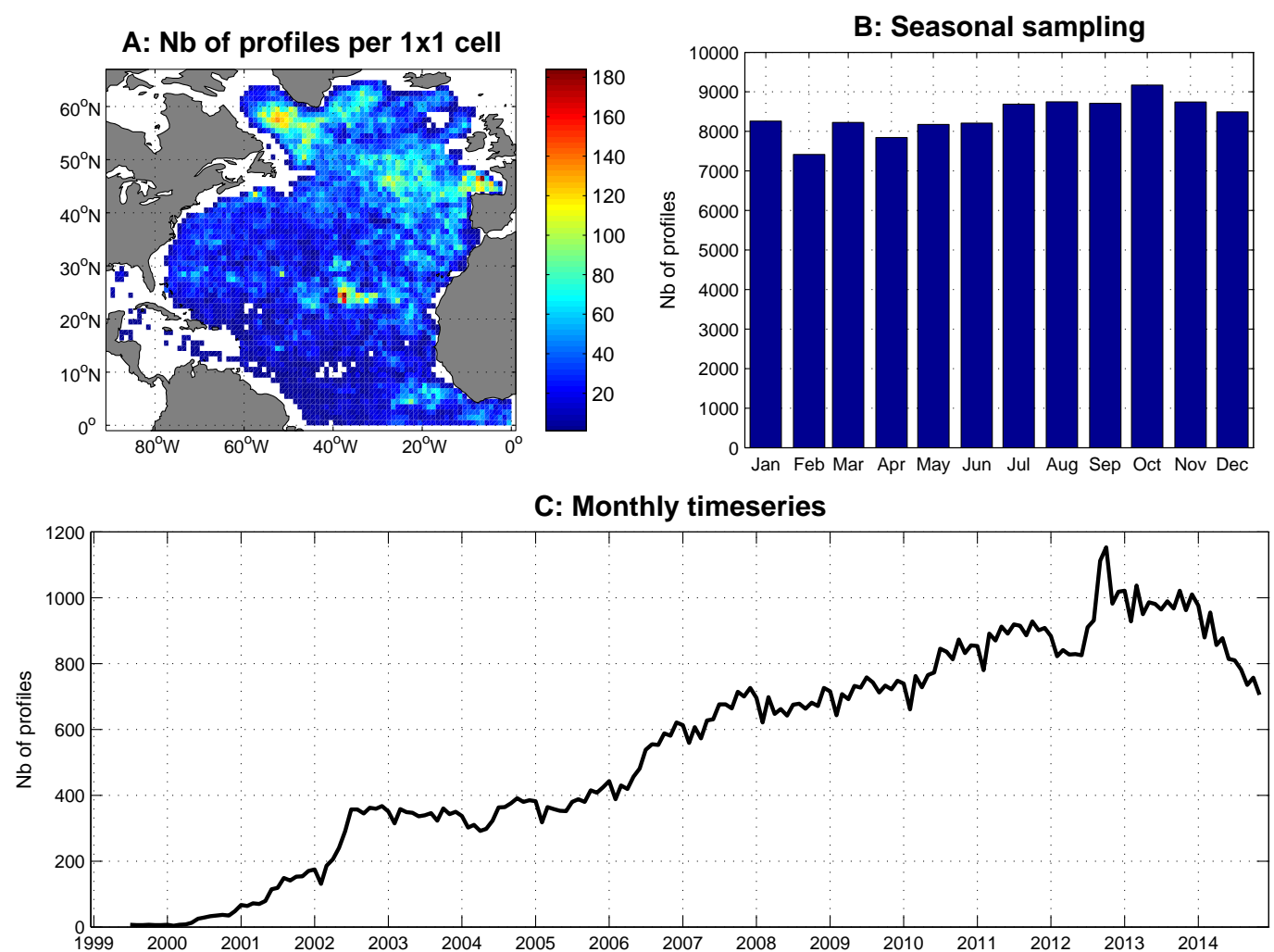

Figure 1: Spatio/temporal sampling of the 100,684 temperature profiles in the Argo dataset used in this study. 

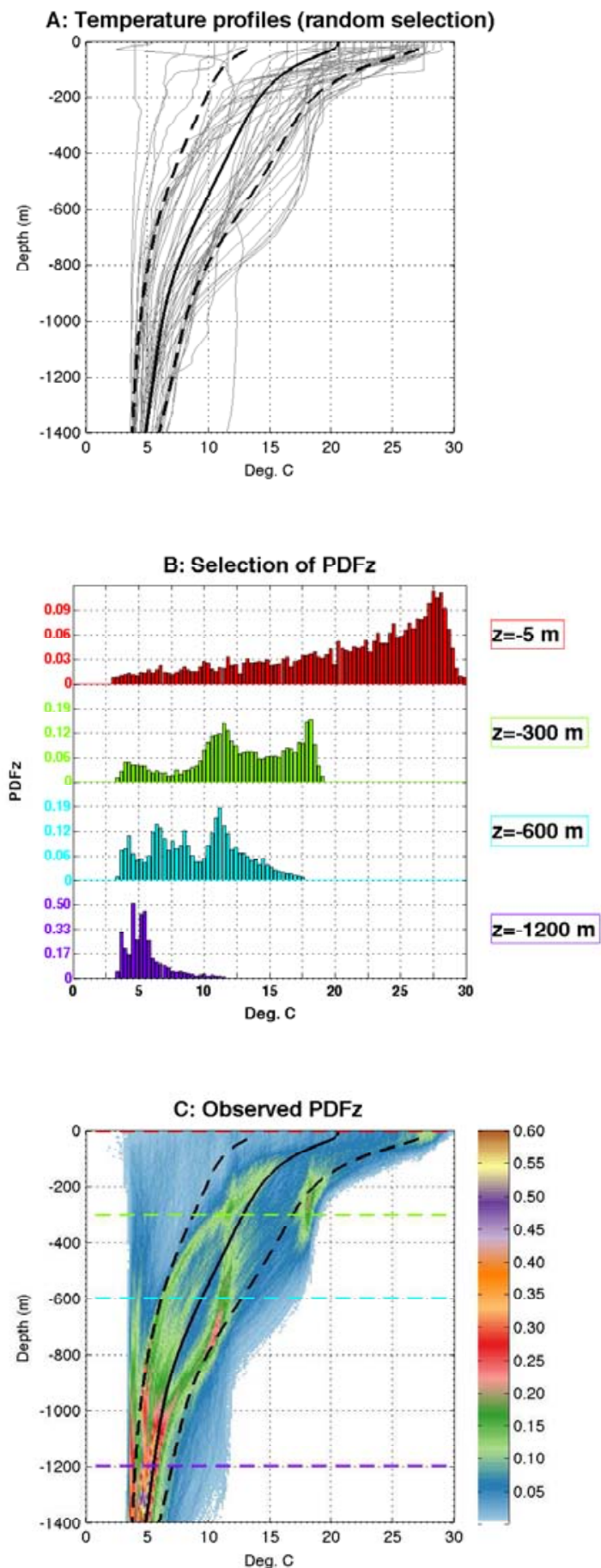

Figure 2: Plot A: 50 profiles randomly drawn out of the Argo collection. Plot B: Sample of PDFz at depth levels: 5, 300, 600 and $1200 \mathrm{~m}$. Plot C: All observed PDFz, i.e. the PDF of temperature at each vertical levels, here computed using the entire collection. The colorscale is the PDF value so that the integral of one PDFz goes to 1 . On plots $\mathrm{A}$ and $\mathrm{C}$ are superimposed in black the mean (plain) and mean \pm one standard deviation (dashed) at each depth. On plot C, horizontal dashed lines indicate plot $\mathrm{B}$ selection of PDFz. 


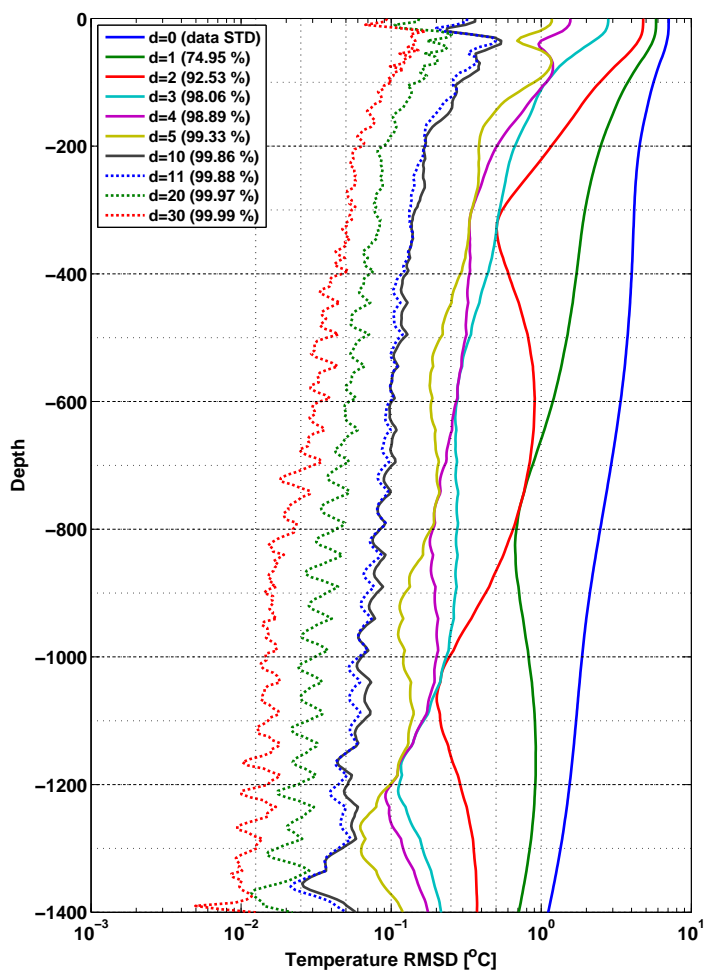

Figure 3: Centered Root Mean Squared Difference between the reconstructed and original datasets of temperature for an increasing number of dimensions used during dimensionality reduction with PCA. The plot can be read as follows: it takes 11 reduced-dimensions (black curve) to compress the dataset in a way that the RMSD is lower than $0.5^{\circ} \mathrm{C}$ throughout the water column. Vertical tick marks are centered at $0.0125,0.025,0.05,0.1,0.25,0.5$ and $1{ }^{\circ} \mathrm{C}$. 


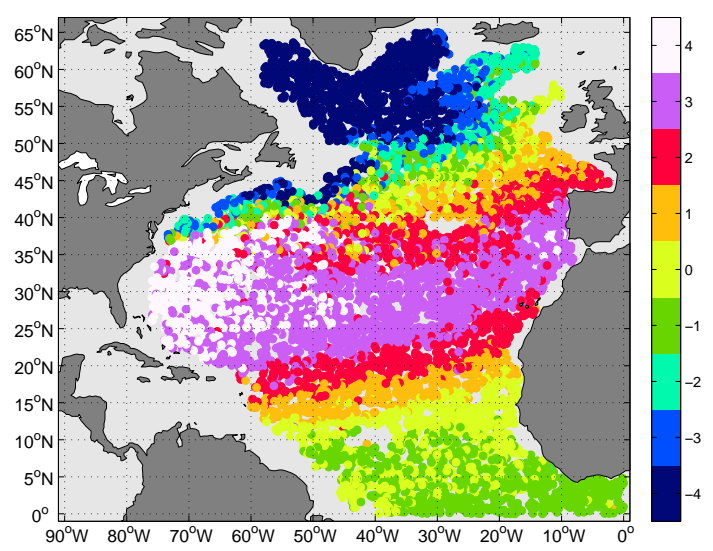

Figure 4: Horizontal distribution of the $0-1400 \mathrm{~m}$ vertical mean temperature, plotted as anomalies with regard to the domain average (which is $9^{\circ} C$ ). We want to investigate the vertical structure of heat anomalies leading to such pattern. Note that only $7.5 \%$ of the profiles have been used to generate this plot (roughly 10 profiles per $2 \times 2$ grid cell) in order to limit overlapping dot pixels. 

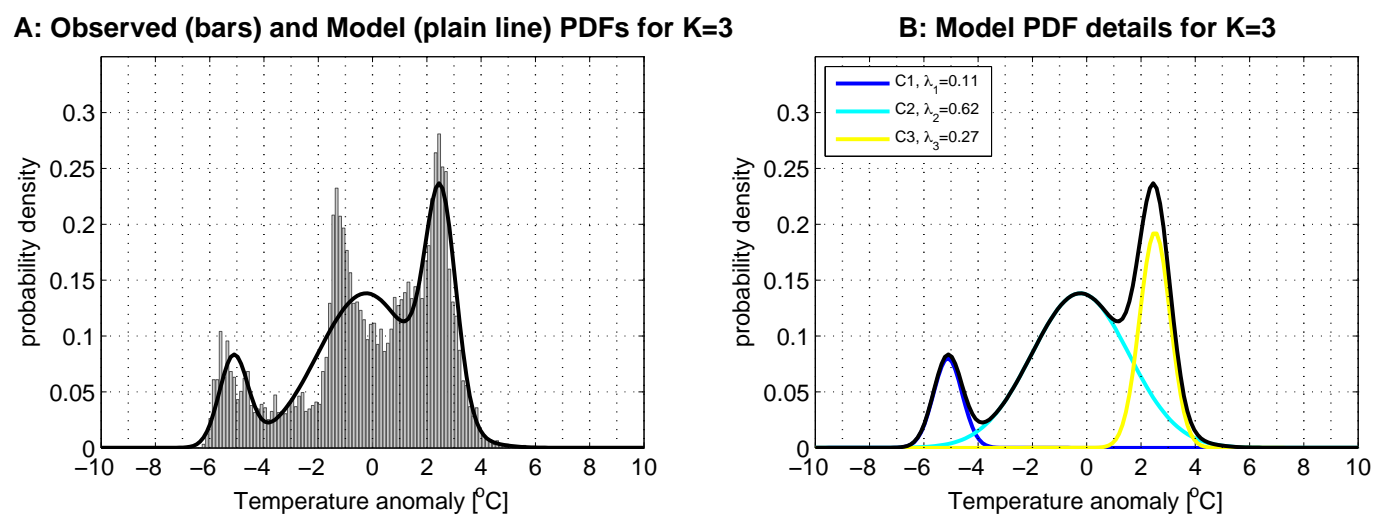

C: Observed (bars) and Model (plain line) PDFs for $K=5$
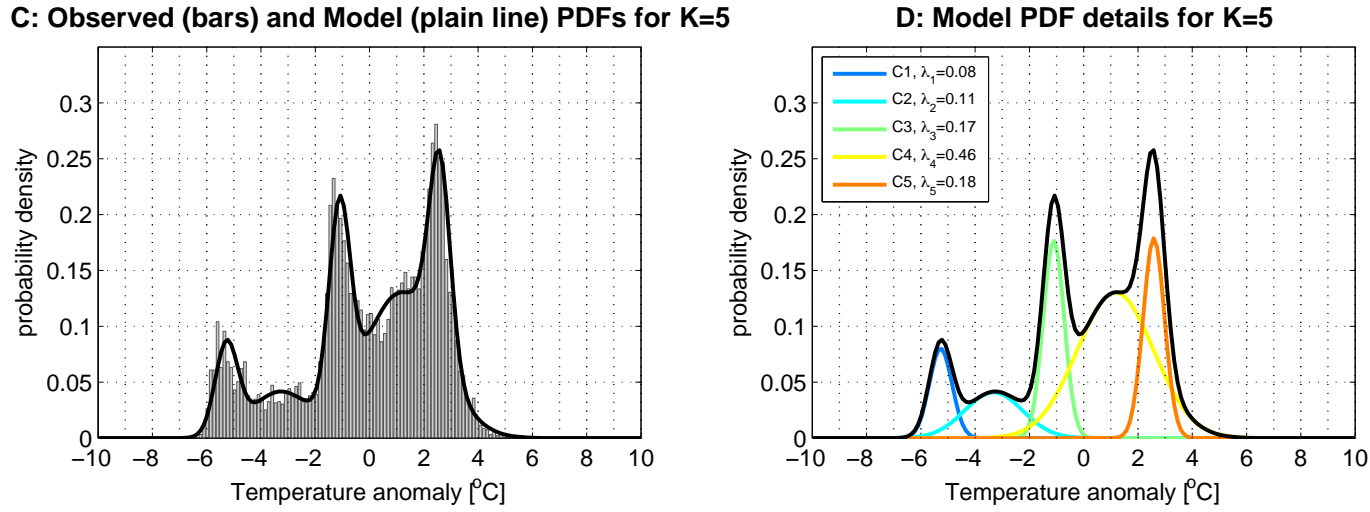

Figure 5: Left plots: gray bars are the observed PDF of the uni-dimensional dataset represented Fig.4 and thick black lines are the GMM PDFs for top: $K=3$ components and bottom: $K=5$ components. Right plots: Decomposition of GMM PDFs into their 3 and 5 components. Colored PDFs are the prior weighted activations of each component. Three distinct modes are clearly visible in the dataset (at the extreme and center of the complete PDF) and are nicely captured by GMM. 


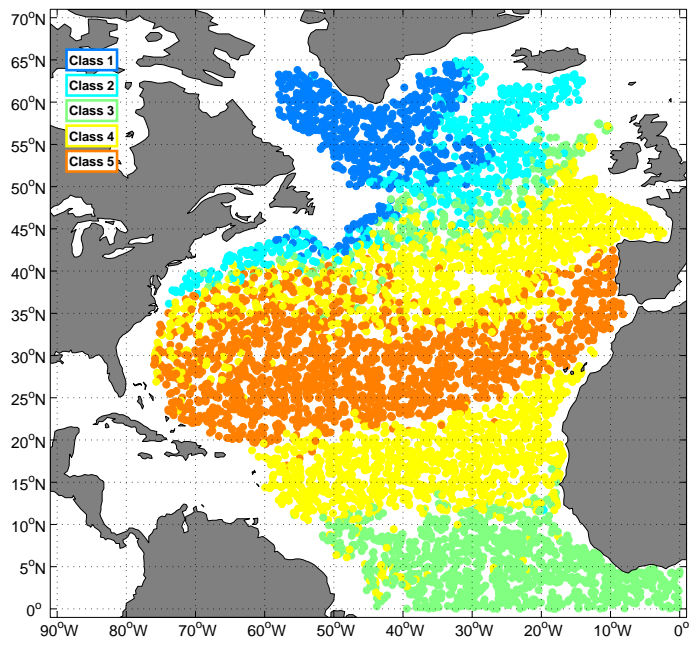

Figure 6: Map of profiles color-coded with the class they have been attributed by Eq.(6) for the uni-dimensional analysis of vertical mean temperature using a GMM with $K=5$. Class color-codes are similar to those from Fig.5-D. 

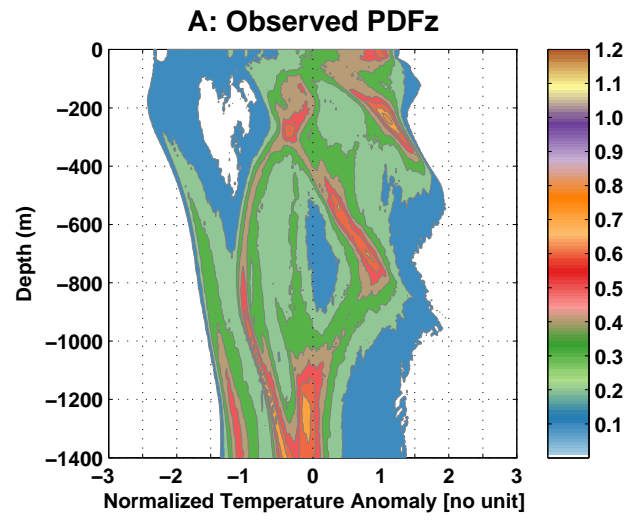

C: Model vs Observed PDFz

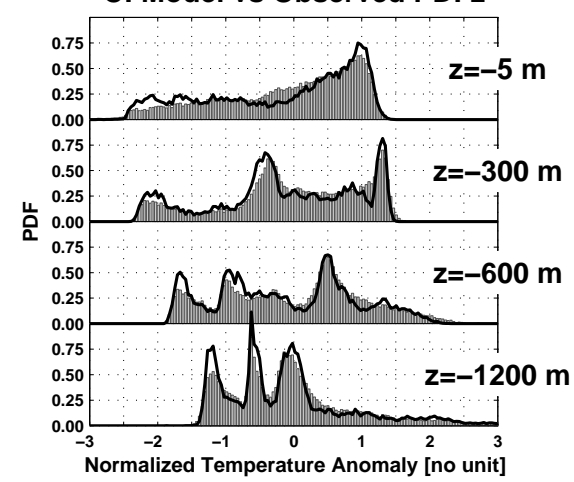

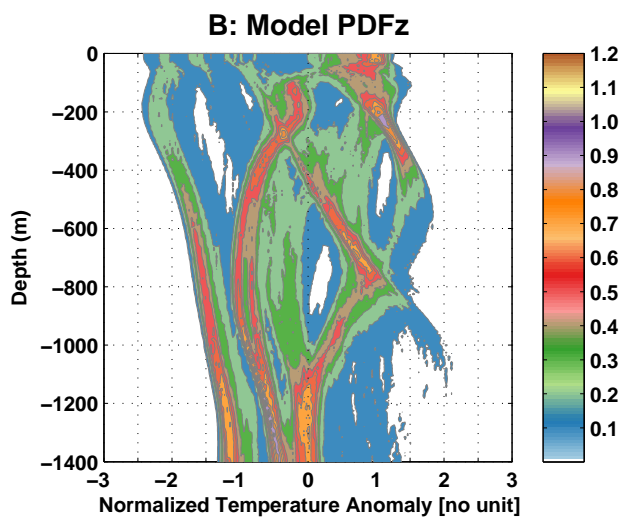

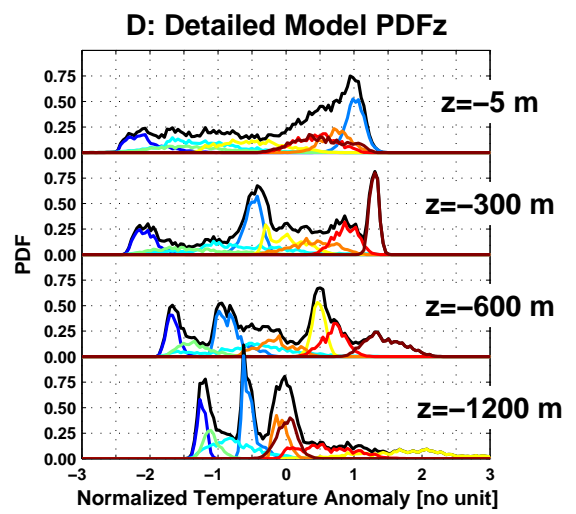

Figure 7: Plot A: Observed PDFz (PDF at each vertical levels) of profiles from the normalized (centered and standardized) temperature dataset. Plot B: Model PDFz from the prior weighted sum of class PDFz (shown in Fig.10). Plot C: Observed (gray bars) and model (thick black line) PDFz for selected depth levels: 5, 300, 600 and 1200m. Plot D: Decomposition of the model PDFz for the same selected depth levels. Colored lines are the prior weighted activations of each class, following the color convention used Fig.9. 


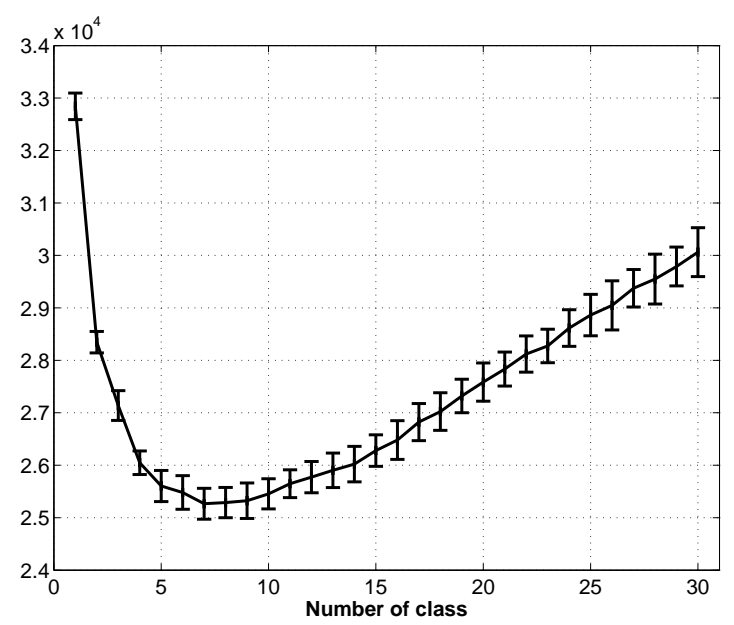

Figure 8: Ensemble mean and spread ( \pm one standard deviation) of the Bayesian information criteria (BIC) for an increasing number of GMM classes K. The ensemble was computed using 50 members of 900 independent profiles with Eq.(4). 

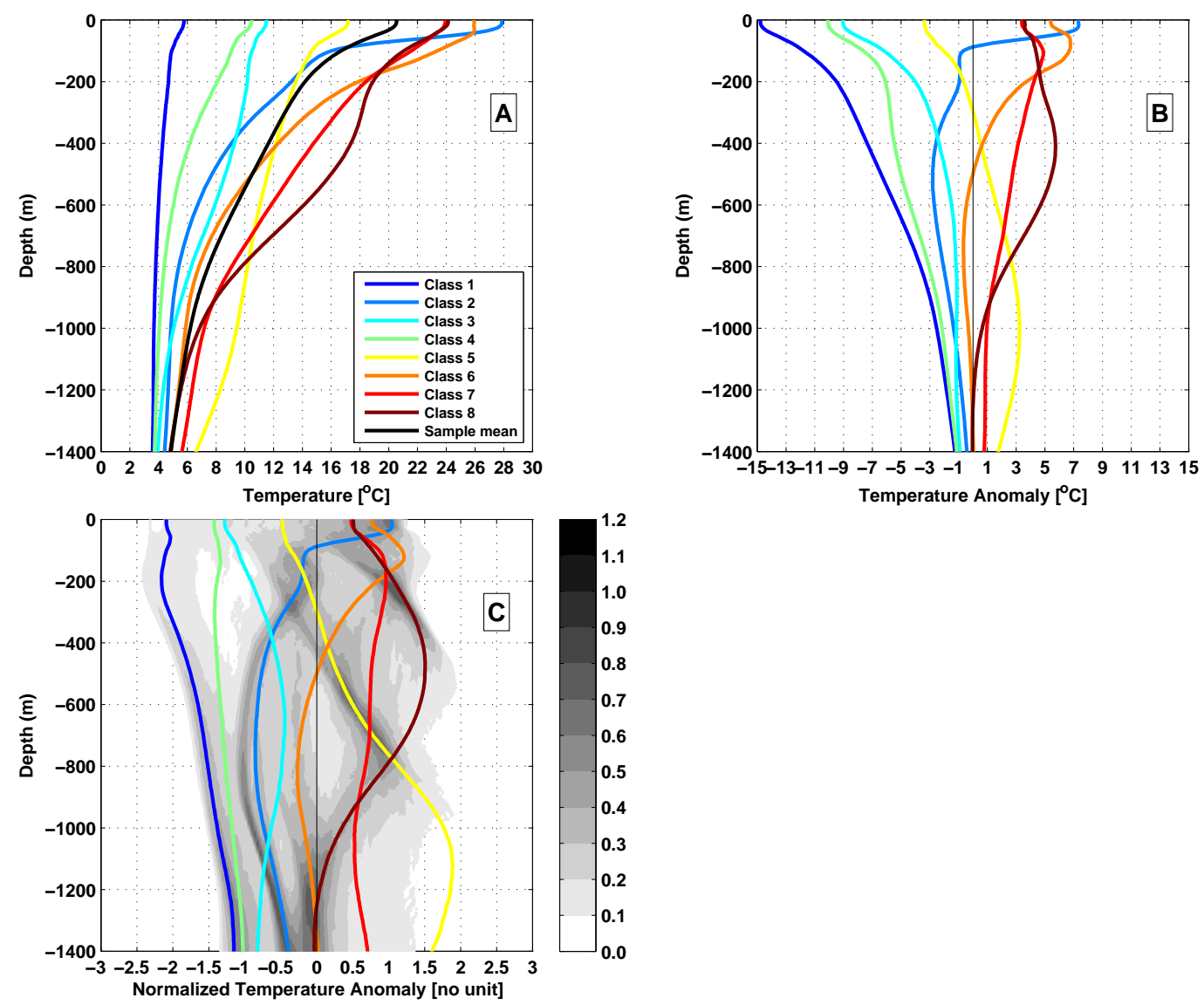

Figure 9: Activation-weighted median profiles for each classes. Plot A: with real temperature profiles (the sample mean reference profile is in black), plot B: with profiles of temperature anomalies (centered dataset) and plot $\mathrm{C}$ with profiles of normalized temperature anomalies (level centered/standardized dataset). Grey shading in plot C is the observed PDFz of the dataset (reproduced from Fig. 7-A). 

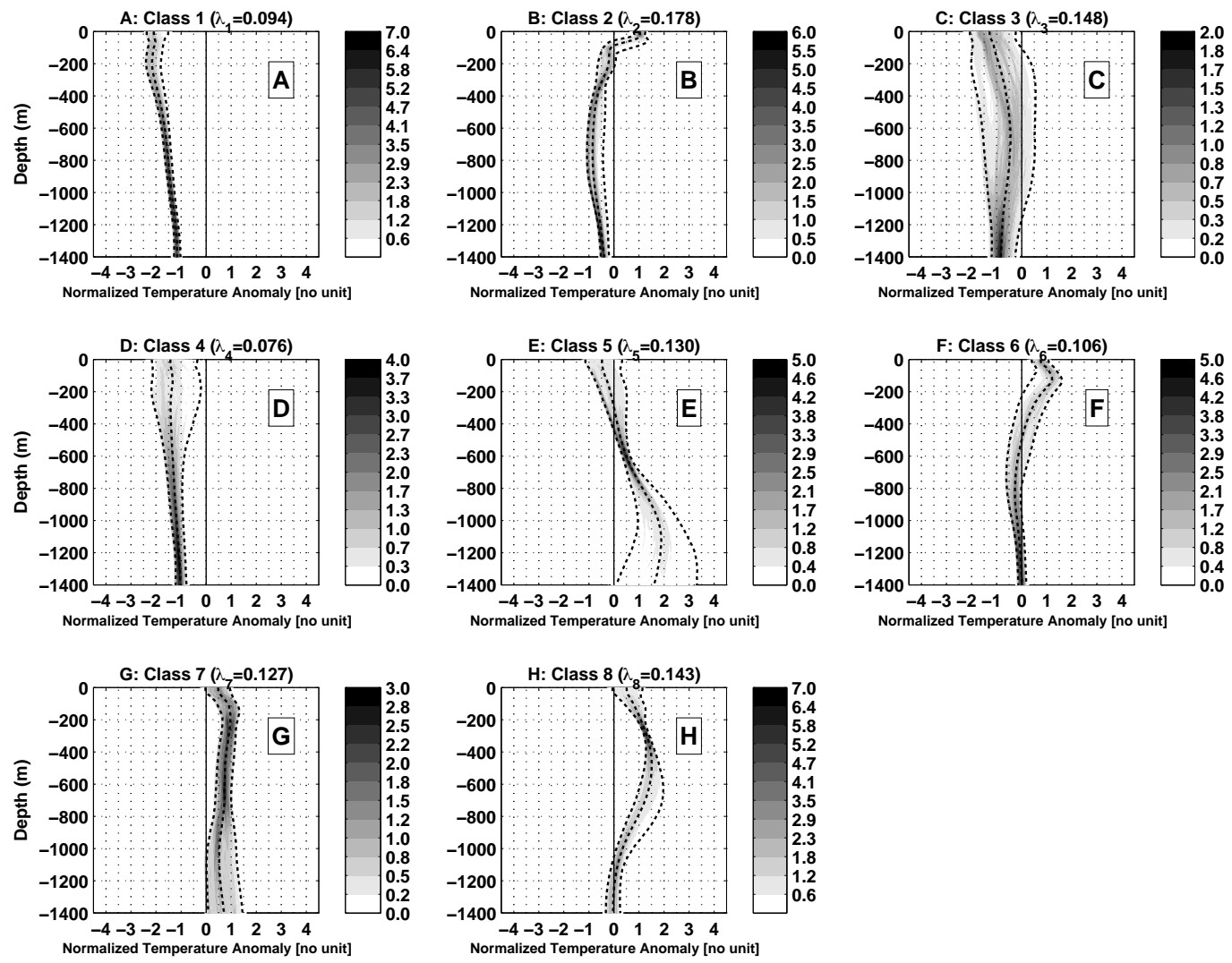

Figure 10: Normalized temperature anomaly for each class as a function of depth: black dashed lines are the 5, 50 and $95 \%$ percentiles of the class (note that the $50 \%$ percentile is the median profile plotted in Fig.9). Grey shading is the corresponding $\mathrm{PDFz}$ for each class temperature anomalies. Also given in the plot title are the prior values $\lambda_{k}$ from Eq.(A.3) of the class. Statistics were computed using temperature data weighted by activations. 


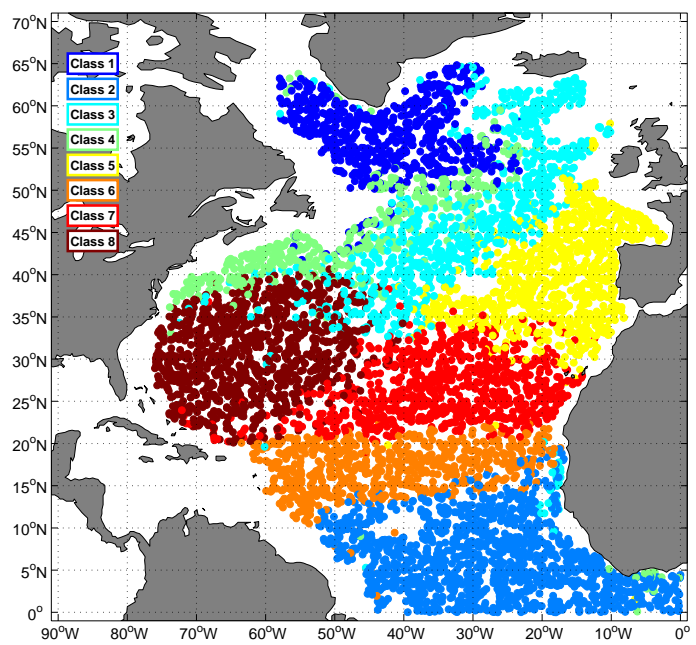

Figure 11: Map of profiles color-coded with the class they have been attributed to with Eq.(6) by the classification of temperature profiles using a GMM with $K=8$. Only profiles with a label metric higher than $90 \%$ (Eq.7) with a limit of 10 profiles per $2 \times 2$ grid cell have been used in order to limit overlapping dot pixels. 

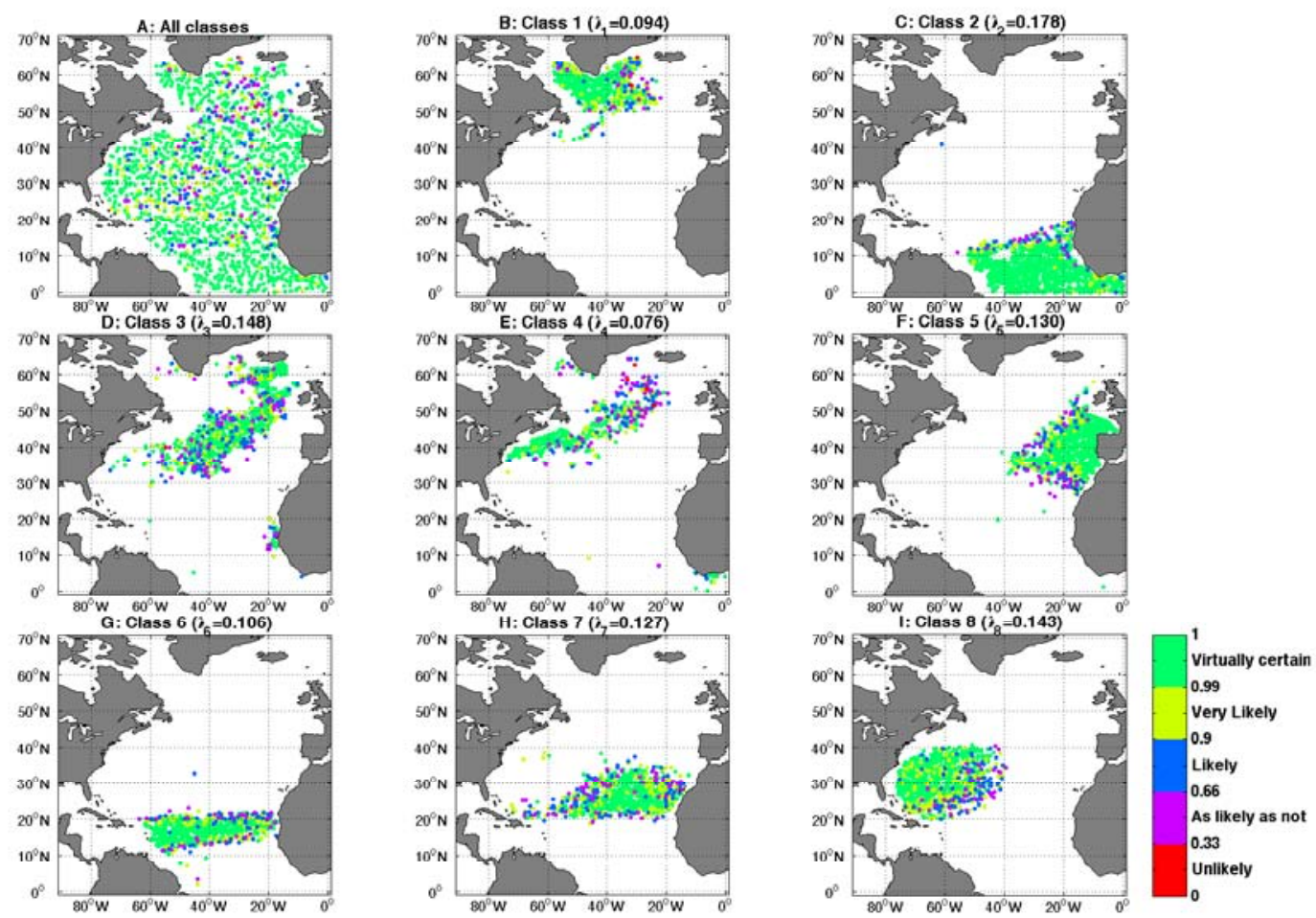

Figure 12: Map of profiles color-coded with their label metric from Eq.(7) for the classification of temperature profiles using a GMM with $K=8$. See how labels are less robust along the edges of classes shown in Fig.11. Map A is for all profiles while maps B to I are for profiles attributed to class 1 to 8 respectively. No more than 10 profiles per $2 \times 2$ grid cell have been plotted in order to limit overlapping dot pixels. 

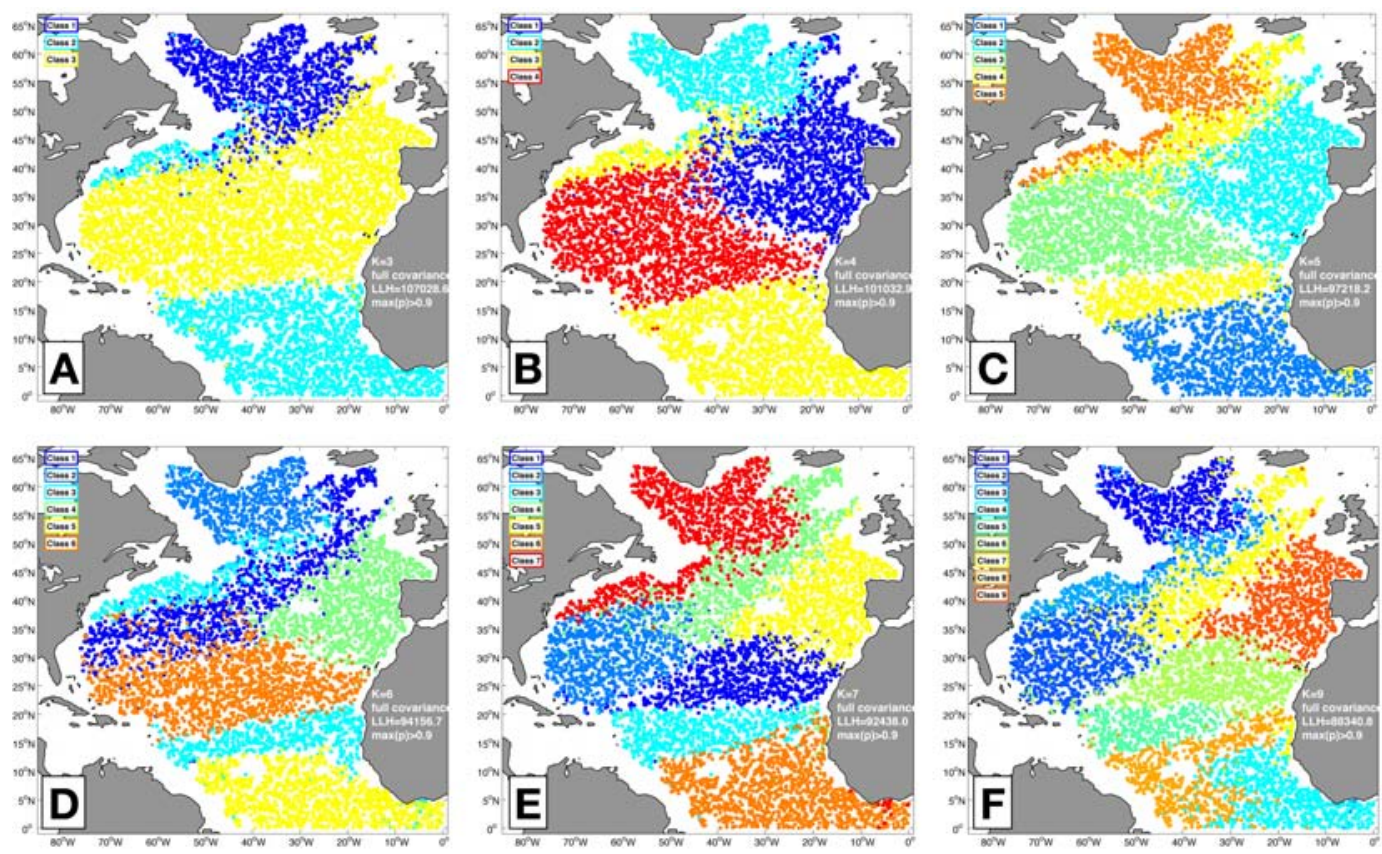

Figure B.13: Map of labels obtained with different number of classes $K$. Map A corresponds to $K=3$, map B to $K=4$, map C to $K=5$, map D to $K=6$, map E to $K=7$ and map F to $K=9(K=8$ is shown in Fig. 11$)$. 

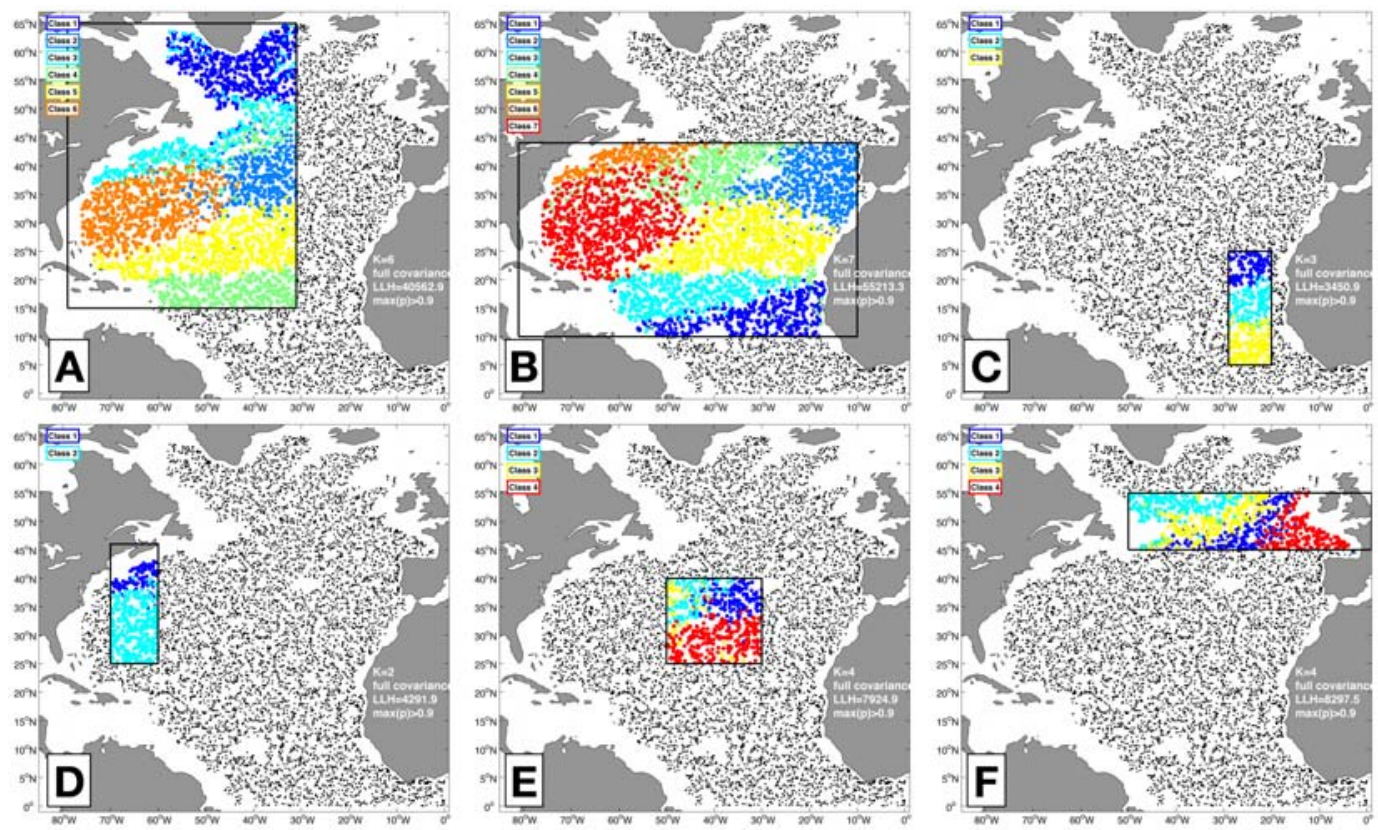

Figure B.14: Sensitivity of the classification to the choice of the domain. On each maps, the black box indicates the domain used to train a new GMM, and profiles are color-coded with the class they have been attributed to. Profiles not used in the GMM training are simply shown as black dots. Note that class colors are arbitrary and only the relative distribution of classes is to be compared with the reference case shown in Fig.11. 


\section{List of Tables}

1 Summary of the different parameters and variables used in a GMM analysis. In practice, the optimized mean and covariance of the $k^{\text {th }}$ classes are computed in the reduced $d$-dimensional space. . . . . . . . . . . . . . . . . . . . . . .

2 This table provides for each of the $K=8$ classes of the reference GMM analysis: the prior values and the dataset fraction of profiles attributed to each class (first and second lines). From the third to seventh lines are given the distribution by class of the labeling metric (Eq.7). First table column provides similar statistics for the entire dataset. The table can be read as follow: $15 \%$ of the dataset can be attributed to class $3,78 \%$ of the class 1 profiles are virtually certain to belong to class 1 given this GMM. . . . . . . . . . . . . . . . . 33 


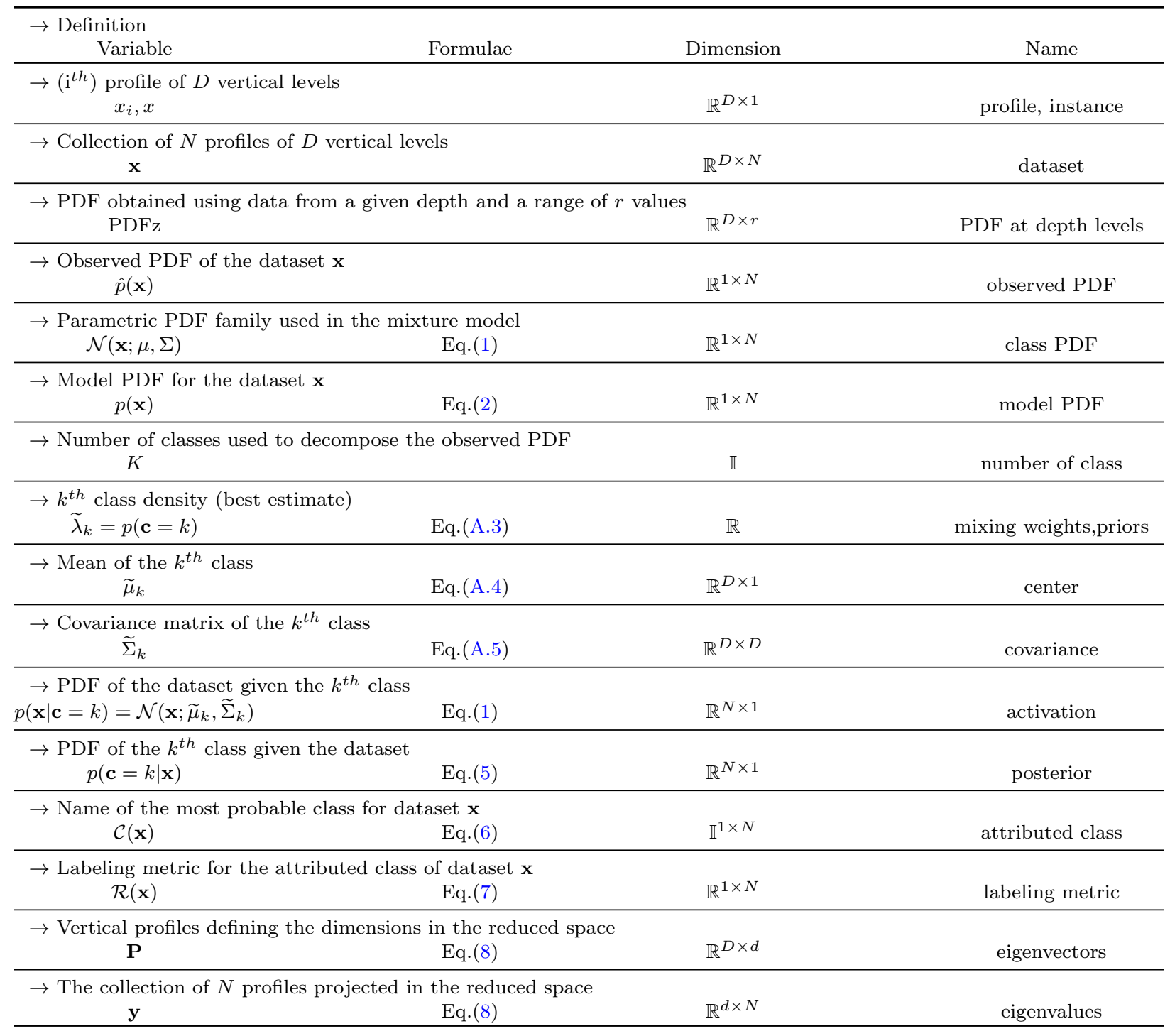

Table 1: Summary of the different parameters and variables used in a GMM analysis. In practice, the optimized mean and covariance of the $k^{t h}$ classes are computed in the reduced $d$-dimensional space. 


\begin{tabular}{|c|c|c|c|c|c|c|c|c|c|}
\hline & All & Class 1 & Class 2 & Class 3 & Class 4 & Class 5 & Class 6 & Class 7 & Class 8 \\
\hline$\lambda_{k}$ Priors & 100 & 9 & 18 & 15 & 8 & 13 & 11 & 13 & 14 \\
\hline Dataset fraction & 100 & 10 & 14 & 15 & 8 & 14 & 9 & 14 & 15 \\
\hline $\begin{array}{c}\text { Unlikely } \\
(0.00<\mathcal{R}<0.33)\end{array}$ & 0 & 0 & 0 & 0 & 0 & 0 & 0 & 0 & 0 \\
\hline $\begin{array}{l}\text { About as likely as not } \\
\quad(0.33<\mathcal{R}<0.66)\end{array}$ & 5 & 3 & 1 & 7 & 10 & 5 & 4 & 6 & 4 \\
\hline $\begin{array}{c}\text { Likely } \\
(0.66<\mathcal{R}<0.90)\end{array}$ & 8 & 6 & 2 & 10 & 13 & 7 & 7 & 10 & 8 \\
\hline $\begin{array}{c}\text { Very Likely } \\
(0.90<\mathcal{R}<0.99)\end{array}$ & 13 & 13 & 4 & 13 & 17 & 10 & 14 & 16 & 17 \\
\hline $\begin{array}{l}\text { Virtually certain } \\
(0.99<\mathcal{R}<1.00)\end{array}$ & 75 & 78 & 92 & 69 & 60 & 78 & 75 & 68 & 72 \\
\hline
\end{tabular}

Table 2: This table provides for each of the $K=8$ classes of the reference GMM analysis: the prior values and the dataset fraction of profiles attributed to each class (first and second lines). From the third to seventh lines are given the distribution by class of the labeling metric (Eq.7). First table column provides similar statistics for the entire dataset. The table can be read as follow: $15 \%$ of the dataset can be attributed to class $3,78 \%$ of the class 1 profiles are virtually certain to belong to class 1 given this GMM. 